\title{
Selective recruitment of non-classical monocytes promotes skeletal muscle repair
}

Authors: Cheryl L. San Emeterio ${ }^{\dagger, 1}$, Claire E. Olingy ${ }^{\dagger, 1}$, YihsuanChu ${ }^{1}$, and Edward A. Botchwey ${ }^{*}, 1$

${ }^{\dagger}$ C.L.S.E and C.E.O. contributed equally to this study and are designated co-first author

\section{Affiliations:}

${ }^{1}$ Wallace H. Coulter Department of Biomedical Engineering; Georgia Institute of Technology and Emory University; Atlanta, GA 30332, USA

\section{*Corresponding Author Contact Information:}

Edward Botchwey

Department of Biomedical Engineering

Georgia Institute of Technology

315 Ferst Drive

Atlanta, Ga 30332

Email: edward.botchwey@bme.gatech.edu

Phone: (404) 385-5058

Fax: (404) 894-4243

Abbreviated Title: Non-classical Monocytes Promote Muscle Repair 


\begin{abstract}
$\underline{\text { Abstract }}$
Regeneration of traumatic defects in skeletal muscle requires the synchronized behavior of multiple cells that participate in repair. The inflammatory cascade that is rapidly initiated after injury serves as a powerful node at which to guide the progression of healing and influence tissue repair. Here, we examine therole thatmyeloid cells play in the healing of traumatic skeletal muscle injury, and leverage their pro-regenerative functionsusing local delivery of the immunomodulatory small molecule FTY720. We demonstrate that increasing the frequency of non-classical monocytes in inflamed musclecoincides with increased numbers of CD206+ alternativelyactivated macrophages. Animals treated with immunomodulatory materials had greaterdefect closure and more vascularization in the acute phases of injury. In the later stagesof repair, during which parenchymal tissue growth occurs,we observed improved regeneration of muscle fibers and decreased fibrotic tissue following localization of pro-regenerative inflammation. These results highlight non-classical monocytes as a noveltherapeutic targetto improve the regenerative outcome after traumatic skeletal muscle injury.
\end{abstract}




\section{Introduction}

Recovery of traumatic skeletal muscle injury relies on precise regulation of the inflammatory cascade that is initiated immediately after injury. While muscle progenitor cells called satellite cells directly differentiate into muscle fibers to regenerate injuredskeletal muscle[1], their success is dependent on supportive cues from innate immune cells, such as monocytes and macrophages, that are acutely recruited to damaged tissue and remain until repair is complete[2-4]. Monocytes and macrophages are phagocytes that are highly sensitive to inputs from their microenvironment and can synthesize this information to direct healing processes[5, 6]. In vivo, monocyte subsets appear sequentially after injury, with classical, inflammatory monocytes accumulating in the muscle after the onset of injury and peaking at around one day post-injury[3]. Non-classical, anti-inflammatory monocytes numbers steadily rise thereafter, and return to baseline once regeneration is complete[3].Monocyte recruitment following the onset of injury is crucial for repair, asmultiplestudies have shownthat depletion of circulating monocytes before toxin-induced muscle injury results in incompletely healed, fibrotic muscle[2-4].

Following extravasation from blood, recruited monocytes may transiently remain monocytes[7] or differentiate into macrophages that persist through the resolution of inflammation [3, 8, 9]. Macrophages designated asclassicalinflammatory “M1" macrophages predominateearly afterinjury, while alternatively-activated “M2” macrophages(AAMs) orchestratethe later phases of tissue repair. Inflammatory macrophages respond to tissue damage signals and upregulate genes associated withalarmins and acute inflammatory-phase proteins[10]. Later during the inflammatory response, AAMs are a potent source of insulin-like growth factor (IGF-1) required for muscle regeneration[11] and highly express genes encoding extracellular matrix (ECM) and ECM remodeling proteins[10].Though some resting tissues such 
as the lung, liver, and brain have macrophage populations that are seeded embryonically[12, 13], skeletal muscle possesses very few macrophagesduringhomeostasis[3]. Upon inflammatoryinsult,circulating classical monocytes are recruited to muscle injury and substantially contribute to the wound macrophage pool[3]. However, whether non-classical monocytes are directly recruited to volumetricskeletal muscle injury or play a role in repair remains unknown.

Knowledge of how differentmyeloid populations affect tissue micro-compartments within injured muscle is especially important in a volumetric defect context in which all tissue architecture must be regenerated de novo. Deposition of new ECM provides scaffolding for nascentvasculature[14], and AAMs have been shown to control collagen fibril formation in regenerating skin[15] and promote ECM deposition by fibroblasts[16].However, the unchecked activity of AAM in chronic muscle inflammationcan perpetuate fibrosis[17], which impairs restoration of native mechanical properties[18]. Muscle tissue must also be revascularized in order to provide necessary oxygen, nutrients, and metabolic waste removal. AAMspromote angiogenesis[19, 20] and arteriogenesis[21], distinct processes that regulate post-injury vascular remodeling. Additionally, failure to reinnervate skeletal muscle after injury results in muscle fiber atrophy[22]that significantly impairs motor function. Increasing AAMs in peripheral nerve transectionsby hydrogel-based delivery of IL-4 enhances both Schwann cell infiltration and axonal growth[23].Consequently, biomaterial-based strategies that increase the number of AAMs within volumetric muscle loss are a promising approach to enhance endogenous programs of muscle repair.

Sphingosine-1-phosphate (S1P) is a pleiotropic bioactive signaling lipid that is produced endogenously by red blood cells, platelets, and endothelial cells[24]. S1P activates signaling 
through five known G protein-coupled receptors, S1PR1-5, which are differentially expressed in almost every cell type.We have previously demonstrated that non-classicalanti-inflammatory $\mathrm{Ly6C}^{\mathrm{lo}}$ monocytes express relatively high levels of S1PR3, which they leverage to exert proregenerative effects in vivo[25]. Local delivery of FTY720, a small molecule agonist of S1P1 and S1PR3-5, selectively recruits non-classical Ly6C ${ }^{\text {lo }}$ monocytes upon local delivery to inflamed skin injuries and promotes arteriogenicmicrovascular growth in an S1PR3-dependent manner. Release of FTY720 from polymer nanofibers to mandibular bone defects increases the frequency of AAMs, promotes re-vascularization, and facilitates boney ingrowth[26].Thus, S1PR3has emerged as a therapeutic target that can orchestrate the inflammatory response and subsequent healing of musculoskeletal injuries.

In this work, we explore the role of specific monocyte and macrophage populations after volumetric muscle loss. Using a novel model of volumetric muscle injury within the murine spinotrapezius muscle, we demonstrate that biomaterial-based delivery of FTY720 increases anti-inflammatory Ly6C ${ }^{\text {lo }}$ monocytes and amplifies the number of CD206+ AAMs. The localization of pro-regenerative inflammation in the injured skeletal muscle increases the kinetics of wound healing, leading to earliervascularization andcollagen deposition.Additionally, we observed improved regrowth of muscle fibers at later time points and less fibrotic collagen architecture within regenerated tissue. These results indicate that immunomodulatory biomaterials that target specific monocyte populations can coordinate favorable healing outcomes within volumetric skeletal muscle injuries. 


\section{Materials and Methods}

\section{Film fabrication}

Films were fabricated as previously described [25]. Briefly, 350 mg PLGA (50:50 DLG 5E Evonik Industries) was added to $2 \mathrm{ml}$ dichloromethane in a glass scintillation vial and vortexed onhigh-speed for 30 minutes. For drug loaded films, 1.75 mg of FTY720 (Cayman Chemical) was added at a 1:200 drug:polymer weight ratio, and polymer solution was vortexed until completely incorporated. The polymer solutions were then poured into Teflon-coated petri dishes and dried at $-20^{\circ} \mathrm{C}$ for 7 days. Films were lyophilized overnight before implantation to remove any traces of solvent.

\section{Spinotrapezius volumetric muscle loss model}

All animal procedures were conducted according to protocols approved by the Georgia Institute of Technology Institutional Animal Care and Use Committee. Male C57BL/6J (The Jackson Laboratory) or B6.129P-Cx3cr1tm1Litt/J mice $\left(\mathrm{CX} 3 \mathrm{CR} 1^{\mathrm{GFP} /+}\right.$ ) (The Jackson Laboratory) of age 8-12 weeks old were used for all animal studies. A 1mm full thickness defect in the spinotrapezius muscle was created as follows. The dorsum of the mouse was shaved and hair removal cream applied to completely remove hair. Skin was sterilized with three washes of 70\% ethanol and chlorhexidine. A longitudinal 1 inch incision (cranial to caudal) was made just after the bony prominence of the shoulder blade. The overlying fascia was dissected away and the spinotrapezius muscle identified. Using flat-tipped tweezers, the edge of the spinotrapezius was lifted up. The muscle was reflected and positioned against a sterile piece of wood. A 1mm biopsy punch was made through the muscle, using the wooden piece as support. The muscle was 
replaced and $1.5 \mathrm{~mm}$ implant placed over the punch defect. The incision was closed with mouse wound clips.

\section{RAW264.7 macrophage and C2C12 myoblast culture and immunohistochemistry}

RAW264.7 macrophages (ATCC) were maintained in growth media (DMEM supplemented with 10\% fetal bovine serum (FBS), $1 \%$ sodium pyruvate, $1 \%$ L-glutamine, $1 \%$

penicillin/streptomycin)according to manufacturer protocols. To generate RAW 264.7 macrophage conditioned media (CM), RAW 264.7 macrophages were treated 18 hours with polarization cytokines. For M(IFN- $\gamma$, LPS) polarization, cells were treated with $1 \mu \mathrm{g} / \mathrm{ml}$ LPS (Sigma) and $20 \mathrm{ng} / \mathrm{ml}$ IFN- $\gamma$ (Peprotech). For M(IL-4) polarization, cells were treated with 10 ng/ml IL-4 (Peprotech). Polarization media was removed after treatment, cells were washed with phosphate-buffered saline (PBS), fresh RAW264.7 media was replaced into the wells. Cells were allowed to condition media for an additional 24 hours.

C2C12 cells (ATCC) were maintained in growth media (DMEM supplemented with 10\% FBS, $1 \%$ sodium pyruvate, $1 \%$ L-glutamine, $1 \%$ penicillin/streptomycin) according to manufacturer protocols. To investigate the effects of polarized macrophage soluble factors on C2C12 myogenic differentiation, C2C12 cells were cultured on fibronectin-coated glass coverslips in 50\% C2C12 differentiation media (DMEM supplemented with 2\% horse serum, 1\% sodium pyruvate, 1\% L-glutamine, 1\% penicillin/streptomycin), and 50\% RAW 264.7 CM for 5 days, with daily media change. At the 5 day timepoint, cells were fixed in $4 \%$ paraformaldehyde (PFA), permeabilized with $0.1 \%$ Triton-X, and blocked using $5 \%$ goat serum for 1 hour. Cells were stained with myosin heavy chain antibody (MF-20, DSHB) overnight at $4^{\circ} \mathrm{C}$ in humidity chamber. Cells were washed, then stained with Dylight 488-conjugated anti-chicken antibody for 
1 hour at room temperature. Cells were washed, counterstained with DAPI, then overturned onto glass slides with Vectashield mounting media (Vector Labs). Cells were imaged on a Zeiss LSM 700 confocal microscope.A total of 12 field of views $(640.2 \mu \mathrm{m} \times 640.2 \mu \mathrm{m})$ were used for histomorphometric analysis of myosin heavy chain-positive cells.

\section{Labeling of blood Ly6C $C^{l o}$ monocytes in CX3CR1 ${ }^{G F P /+}$ mice}

In order to visualize cells within injured muscle originating as circulating $\mathrm{Ly}_{6 \mathrm{C}} \mathrm{C}^{\mathrm{lo}}$ monocytes, CX3CR $1^{\mathrm{GFP}} /+$ mice were administered Fluoresbrite ${ }^{\circledR}$ Polychromatic Red latex beads $(0.5 \mu \mathrm{m}$, Polysciences) intravenously 1 day prior to surgery via jugular vein injection. Prior to injection, latex beads were diluted 1:25 in sterile saline as previously described[27].Labeling was confirmed by retro-orbital blood draw immediately prior to surgery (1 day after bead administration) and subsequent flow cytometry analysis.

\section{Flow cytometry}

To collect blood (days 0, 3 and 7) and tissue for flow cytometry analysis (days 1, 3, and 7), mice were euthanized via $\mathrm{CO}_{2}$ asphyxiation. Blood was then collected via cardiac puncture. Red blood cells were lysed in ammonium chloride (1 part blood, 9 parts ammonium chloride) prior to immunostaining for flow cytometry.For characterization of myeloid cell composition in spinotrapezius muscles without an implant, a $6 \mathrm{~mm}$ biopsy punch of muscle tissue centered on the defect was taken. For studies with an implant (unloaded or FTY720-loaded), the entire spinotrapezius muscle was collected.Spinotrapezius muscles were harvested and digested in collagenase I (Sigma) for 45 minutes at $37^{\circ} \mathrm{C}$. The digested muscles were filtered through a cell strainer to obtain a single cell suspension. The standard recovery of cells from a $6 \mathrm{~mm}$ biopsy of 
the spinotrapezius muscle is $5501 \pm 1965$ cells/mg tissue (measured in similar, but unrelated studies).Single-cell suspensions from were stained for flow cytometry analysis in 3\% FBS according to standard procedures and analyzed on a FACS AriaIIIu flow cytometer (BD Biosciences). The following antibodies were used for cell phenotyping: BV510-conjugated antiCD11b (BioLegend), PerCP eFluor-710-conjugated anti-CD115 (eBioscience), APC-Cy7 conjugated anti-CD11b (BioLegend), BV421-conjugated anti-CD11c (BioLegend), APC conjugated anti-Ly6C (BioLegend), PE-Cy7 conjugated anti-GR-1 (BioLegend), BV711conjugated anti-CD64 (BioLegend), PE-conjugated anti-MerTK (R\&D Systems), APC-Cy7conjugated anti-Ly6G (BioLegend), PE-Cy7-conjugated anti-CD206 (BioLegend).

\section{Whole mount immunohistochemistry of muscle tissue}

Mice were euthanized 3 or 7 days after surgery via $\mathrm{CO}_{2}$ asphyxiation. For lectin-based labeling of the vasculature in CX3CR1 $1^{\mathrm{GFP} /+}$ mice, Alexa Fluor 568 isolectin IB4 (1mg/mL, Life Technologies) was diluted $(50 \mu \mathrm{L}$ of isolectin, $150 \mu \mathrm{L}$ saline) and administered by jugular vein injection 15 minutes prior to euthanasia. Post-euthanasia, mouse vasculature was perfused with warm saline followed by 4\% PFA until tissues were fixed. The entire spinotrapezius muscle was explanted and permeabilized overnight with $0.2 \%$ saponin, then blocked overnight in $10 \%$ mouse

serum. For immunofluorescence, tissues were incubated at $4^{\circ} \mathrm{C}$ overnight in a solution containing $0.1 \%$ saponin, $5 \%$ mouse serum, $0.5 \%$ bovine serum albumin, and the following conjugated fluorescent antibodies: PE anti-CD31 antibody (1:100 dilution, eBioscience), Alexa Fluor 488 anti-CD206 (1:200 dilution, ABD Serotec), and Alexa Fluor 647 anti-CD68 (1:200 dilution, ABD Serotec). For desminimmunostaining, identical tissue processing procedures were followed and muscles were incubated overnight with Alexa Fluor 488 anti-desmin (1:200 dilution, 
Abcam) alone or in combination with PE anti-CD31 and Alexa Fluor 647 anti-CD206 (1:200 dilution,BioLegend).Followingimmunostaining, tissues were washed 4 times for 30 minutes each, and then mounted in 50/50 glycerol/PBS.

\section{Imaging and quantification of whole mount immunohistochemistry}

Tissues were imaged on a Zeiss LSM 710 NLO confocal with acquisition parameters kept identical across all animals. For collagen imaging, a two-photon chameleon laser (tuned to $\lambda=810 \mathrm{~nm}$ ) and collection range of 380-420nm was utilized with acquisition parameters kept identical across animals. Z-stacks were collected from the beginning to end of collagen signal visible across the entire $x-y$ plane analyzed.Max intensity projections were generated from 3D confocal images for 2D analysis in Zen software (Zeiss). Quantification of vascularization and collagen deposition was performed in ImageJ software (NIH). An elliptical region of interest (1.370 mm high by $1.778 \mathrm{~mm}$ wide) was centered over each defect and the image was thresholded to display only areas of positive fluorescence signal.The number of pixels positive for the fluorescence channel of interest was normalized to the total area of the region of interest.In Figures 1 and 6, defect area was determined by tracing the edges of desmin-positive immunostaining to outline thedesmin-negative area. The defect area was identified as the area that wasdesmin-negative. In Figure 5, the percentage of cell infiltration was determined by tracing the border between lectin- or CD31-positive regions and the avascular zone. The area of this region was measured. A secondary trace between the cellular (CX3CR1+ or CD206+) region and acellular zone was made. The area of this region was measured and subtracted from first measurement to measure the region of cell infiltration into the defect. This value was normalized to the first measurement. In Figure 6, "void area” was measured by tracing the edges of collagen- 
positive signal and identifying void areaas the area that is collagen-negative. 2D fiber diameter was determined by measuring the widest portion of each regenerated muscle fiber (determined based on the morphology of desmin-positive regions) within the defect region from the day $72 \mathrm{D}$ maximum intensity projections.

For 3D analysis in Imaris (Bitplane), 1 crop per animal of 332x332 $\mu$ m was taken from the area adjacent to the defect to show immune cell distribution in the close surrounding tissue. Crops were chosen based on matching anatomical vessel morphology from both CX3CR1 ${ }^{\mathrm{GFP} /+}$ images and the subsequently stained CD206, CD68, and CD31 images.Simultaneous visualization of CX3CR1-GFP cells with immunohistochemical markers is not possible due to leakage of GFP from the cells following permeabilization.Therefore, imaging of CX3CR1-GFP spinotrapezius muscles occurred immediately after perfusion fixation and further immunostaining was performed as described in the previous methods section.Cells co-expressing CD68 and CD206 were identified in Imaris using the surface tool. CD68+ surfaces wereidentified by smoothing with a $1.5 \mu \mathrm{m}$ grain size and an automatic threshold on absolute intensity. Touching objects were split using a seed points diameter of $13.8 \mu \mathrm{m}$. Cells that co-expressed two markers were identified by applying an additional filter to select surfaces with a high fluorescence intensity in the CD206 channel (above 17.7 mean intensity). Vessels were identified in Imarisby drawing a surface on the CD31 fluorescent channel with a $1.66 \mu \mathrm{m}$ grain size, manually-selected threshold value (determined based on each image), and manually-selectedvolume filter to remove small debris. To calculate the distance between CD68+CD206+ cells and the nearest blood vessel, a distance transformation was applied to CD31+ vessel surfaces and the median position of each CD68+CD206+ cell within this plane was recorded. 


\section{Statistical analysis}

Data are presented as mean \pm standard error of the mean (S.E.M.), unless otherwise noted. All statistical analysis was performed in GraphPad Prism software. For pairwise comparisons, unpaired t-test (according to experimental design) with Welch’s correction if variance was significantly different wasused. For grouped analyses, two-way ANOVA with Sidak's post-test was used for multiple comparisons. Unless otherwise noted, $\mathrm{p}<0.05$ was considered statistically significant.

\section{$\underline{\text { Results }}$}

Full-thickness defect in the murine spinotrapezius muscle results in dynamic myeloid cell kinetics both locally and systemically.

In order to better model clinical muscle loss induced by trauma or surgery,we developed a novel model of volumetric muscle loss (VML) in the murine spinotrapezius (Figure 1A).The spinotrapezius muscle is a stabilizing muscle in the mouse dorsumthatis extremely thin (60$200 \mu \mathrm{m})[28,29]$,enabling the use of three-dimensional (3D) confocal microscopy to examine tissue microstructures. A 1mm circular defect in the spinotrapezius muscle heals over the course of 7 days, as observed by desminimmunostaining of muscle fibers(Figure 1B, C). Consequently, removal of a $1 \mathrm{~mm}$ diameter muscle defect is not critical-sized, but enables investigations into the mechanisms, rate, and quality of muscle repair. We examined healing at the defect edge, specifically focusing on granulation tissue formation and myofiber ingrowth. Granulation tissue was defined as tissue lacking desmin+ mature muscle fibers, but including CD31+ and CD206+ immunostaining, asthe proliferative phase of the wound healing response involves angiogenesis 
and the presence of fibroblasts and macrophages, the latter of which can express CD206 and play an active role in repair [5, 30,31]. Highpower images of the defect edge reveals granulation tissue containing CD206+ cells, indicating the presence of AAMs (Figure 1D). CD31 immunostaining reveals vessel regrowth within the granulation tissue, with CD206+ cells interacting closely with nascent vasculature. This revascularization appears to occur before the regrowth of desmin+ muscle fibers at day 7 (Figure 1E, F).

We then investigated the kinetics of myeloid cell trafficking in response to spinotrapezius VML injury. The phenotypic complexity of myeloid cells during inflammation necessitates understanding baseline healing conditions in order to design therapies that leverage their behavior. Blood neutrophils were transiently elevated roughly 5 fold from baseline 3 daysafter VML injury (11.9 $\pm 2.7 \%$ at day 3 vs.2.1 $\pm 0.8 \%$ at day 0 , mean \pm SD as a percentage of all cells), and subsequently decreased (Figure 2A). Blood classical Ly6C ${ }^{\text {hi }}$ monocytes slowlyincreased over 7 days (1.0 $\pm 0.3 \%$ at day 0 compared to $3.8 \pm 1.6 \%$ at day 7$)$,while blood non-classical Ly6C $\mathrm{C}^{\mathrm{lo}}$ monocytes increasedmore rapidly from baseline by 3 days $(2.6 \pm 0.8 \%$ at day 0 to 8.5 $\pm 1.6 \%$ ) and plateaued at 7 days post-injury (Figure 2B). Within injured spinotrapezius muscle tissue, neutrophils peaked at 3 days post-injury ( $0.7 \pm 0.2 \%$ of all cells) and quickly decreased to baseline levels by 7 days post-injury (0.1 $\pm 0.3 \%$ ) (Figure 2C). Ly6C ${ }^{\text {hi }}$ monocytes decreasedfrom days $1(0.7 \pm 1.2 \%)$ to $7(0.3 \pm 0.5 \%)$, while Ly6C $\mathrm{C}^{\mathrm{lo}}$ monocytes peaked at day $3(1.5 \pm 0.9 \%)$ postinjury and were retained at a similar frequency at day 7 (Figure 2D). MerTK and CD64 exclusively identify macrophages (MerTK+CD64+)[32], which steadily increase from day 1 $(0.5 \pm 0.6 \%)$ to day7(3.0 $\pm 1.0 \%)$ (Figure $2 \mathrm{E})$. CD206+ macrophages also similarly increase over time $(0.4 \pm 0.6 \%$ at day 1 to $2.8 \pm 1.0 \%$ at day 7 ) (Figure $2 \mathrm{~F}$, Supplemental Figure $2 \mathrm{~A}$ ). Interestingly, MerTK+CD64+ macrophages comprise a relatively small proportion of all 
CD206+ cells (5.3 $\pm 2.4 \%$ at day 3, and $27.4 \pm 2.8 \%$ at day 7 post-injury) (Supplemental Figure 2B), as analyzed by flow cytometry. Other cells involved in wound healing may express CD206, including fibrocytes[33] and immature dendritic cells[34], but further immunophentoyping of these cells is necessary to identify other CD206+ populations within injured skeletal muscle.Taken together, our kinetic data suggest that neutrophils and Ly6C ${ }^{\text {hi }}$ monocytes accumulate in the acute inflammatory phase of VML injury, while macrophages, particularly CD206+ AAMs, predominate in the later phases of muscle injury and healing.

\section{Non-classical blood monocytes are directly recruited to volumetric muscle injury.}

Intravascular administration of latex (LX) beads can be used to track the recruitment and fate of circulating monocytes[27, 35]. We administered LX beads one day prior to injury in heterozygous CX3CR1 $1^{\mathrm{GFP} /+}$ mice, which constitutively express green fluorescence protein (GFP) under the CX3CR1 promoter (Figure 3A).Circulating $\mathrm{Ly}_{6 \mathrm{C}}{ }^{\mathrm{lo}} \mathrm{CX} 3 \mathrm{CR} 1^{\mathrm{hi}}$ monocytes are selectively labeled with LX beads 1 day after injection (Supplemental Figure 1C, D).During surgery, a 1.5mm poly(lactic-co-glycolic acid) (PLGA) film was placed over the defect (Figure3B) to model biomaterial implantation within VML. Accumulation of LX+ cells in injured skeletal muscle tissue was monitored with confocal microscopy. Both CX3CR $1^{\text {lo }}$ and CX3CR $1^{\text {hi }}$ monocytes were present in spinotrapezius muscle tissue 3 days post-injury (Figure 3C). Interestingly, a high frequency of LX+ cells were also seen in the muscle tissue, indicating that labeled Ly6C ${ }^{\text {lo }}$ monocytes were directly recruited from circulation to the injured tissue (Figure 3C). This phenomenon stands in contrast to studies conducted using a toxin-induced muscle injury modelin which no LX bead-labeled Ly6C $^{\text {lo }}$ monocytes were recruited from blood[3]. High-power 3D images of immunostainedperi-defect muscle tissue at 3days post-injury 
show LX+ cells spatially co-localizing with CD68+CD206+ cells, animmunophenotype indicative of pro-regenerative AAMs (Figure3D, Supplemental Figure 3A, B). These results suggest direct conversion of blood-derived Ly6C $\mathrm{C}^{\mathrm{lo}}$ monocytes into CD206+ macrophages within muscle tissue and provide a novel therapeutic target for regulating the accumulation of proregenerative macrophages in inflamed tissue.

M(IL-4) macrophage conditioned media promotes myogenic differentiation of C2C12 myoblasts.

To more closely probe effects of macrophage phenotype on myogenesis, we conducted an in vitro differentiation assay using C2C12 myoblasts treated for 5 days with conditioned media from polarized RAW264.7 macrophages stimulated with either LPS and IFN- $\gamma$ (M(LPS,

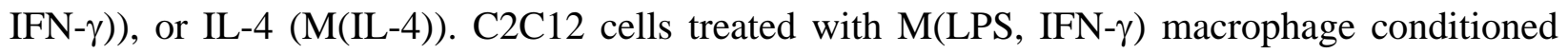
media exhibited no differentiation over the course of 5 days (Figure 3E). Conversely, C2C12 cells treated with $\mathrm{M}(\mathrm{IL}-4)$ macrophage conditioned media exhibited high myogenic differentiation, with the presence of several multinucleated myotubes in the culture. These results indicate that secreted factors from M(IL-4) AAM-likemacrophagessupport myogenesis, and provides further motivation for developing therapeutic strategies that increase this phenotype of macrophage within injured muscle.

Local delivery of FTY720 from polymeric biomaterials increases $L y 6 C^{l o}, C X 3 C R 1^{h i}$ monocytes and CD206+ macrophages in injured muscle tissue.

Previous work in our lab demonstrated that local administration of FTY720 to skin injury increases the frequency of CD206+ macrophages that were derived from blood Ly6C ${ }^{\text {lo }}$ 
monocytes[25]. We sought to determine whether this strategy could be leveraged in VML injury to enhanceaccumulation of CD206+ macrophages within damaged muscle tissue. Implantation of anFTY720-loaded PLGA thin film over the muscle defect increased the frequency of Ly6C ${ }^{\text {lo }}$, but not Ly6C $C^{\text {hi }}$, monocytes compared to PLGA only or no implant controls,as determined by flow cytometry (Figure 4A).After induction of VML injury in CX3CR1 ${ }^{\mathrm{GFP} /+}$ mice, very few CX3CR1GFP+ cells were detected in the uninjured contralateral spinotrapezius muscle (Figure 4B). Qualitative analysis of whole mount tissues shows that on-site delivery of FTY720increased accumulation of CX3CR $1^{\text {hi }}$ cells in the peri-defect muscle tissue (Figure 4C), which is consistent with the flow cytometry data that shows increased Ly6C $\mathrm{C}^{\text {lo }}$ monocyte frequencyfollowing FTY720 treatment, and analysis of blood monocytes shows inverse correlation of surface CX3CR1 and Ly6C expression (Supplemental Figure 1A, B).Ly6C ${ }^{\text {lo }}$ blood monocytes were labeled in CX3CR1 ${ }^{\mathrm{GFP} /+}$ mice with LX beads1 day prior to VML surgeryto track the fate of Ly6C $^{\text {lo }}$ monocytes during FTY720-mediated immunomodulation. Inspection of wholemount confocal images reveals more LX+ beads inthe muscle tissue of FTY720-treated animals and that these LX+ beads were positioned closely around the defect (Figure 4D).

To measure cellular infiltration into the defect, we measured the relative size of the avascular region with high myeloid cellularity (both CX3CR1+ and CD68+CD206+ cells). FTY720-treated animals had a larger percentage of the defect filled with CX3CR1+ cells (Figure 5A, B). We observed a similar, but insignificant, trend following quantification of the proportion of the defect filled with CD68+ and CD206+ cells (Figure 5C, D). Interestingly, we noticed cellular regions that were sparse in CX3CR1+ cells but contained numerous CD68+CD206+ cells. Since we observed conversion of Ly6C ${ }^{\text {lo }}$ monocytes into CD206+ macrophages (Figure 3D), we investigated whether FTY720 also increases the frequency of CD206+ macrophages in 
injured muscle. Animals treated with FTY720 had more CD68+CD206+ macrophages in peridefect tissue 3 days post-injury (Figure 5E, F).Close interactions of macrophages and endothelial cells have beenassociated with enhanced vascular remodeling and arteriogenesis[25, 36].Quantification ofthe distance between CD68+CD206+ macrophages and the nearest CD31+ blood vessel within damaged spinotrapezius muscles revealed that AAMs homed closer to vasculature with FTY720 treatment (Figure5C,G).

\section{${ }^{2 y 6 C^{l o}}$ monocytes / CD206+ macrophages promote skeletal muscle healing after traumatic injury.}

To investigate whether FTY720-induced perivascular accumulation of CD206+ macrophages was associated with repair of injured skeletal muscle, we assessed wound healing parameters. By 3days post-injury, local delivery of FTY720 was able to induce increased revascularization of the defect region (Figure6A,B), as quantified by CD31 immunostaining. Interestingly, the amount of revascularization in FTY720-treated animals was similar to animals with no implant (Figure 6B, Supplemental Table 1), indicating that PLGA may impair revascularization. Increased collagen deposition within the defect area,as measured by imaging the second harmonic generation (SHG) signal, was observed in FTY720-treated animals (Figure6C,D) coinciding with a significantly decreased non-collagenous void area. Regrowth of mature desmin+ muscle fibers was more prominent in muscles treated with FTY720 and these animals demonstrated atrend of reduced defect area at 3 days post-injury (Figure 6E, F). Defects left untreated (no implant) had a lower average defect area than PLGA-treated mice and a lower average defect area than FTY720-treated mice (Figure 6F, Supplemental Table 1), indicating that PLGA may impair defect closure. 
At day 7 post-injury, injured muscles were imaged with two-photon confocal microscopy for immunostaineddesminand SHG collagen signal. We rendered the 3D images in Imaris ${ }^{\mathrm{TM}}$ to generate quantitative volumes of desmin and collagen signal. FTY720-treated animals displayed a lower volume ratio of collagen to desmincompared to PLGA animals (Figure 7A, B), indicating less interstitial fibrosis. Furthermore, control animals displayed highly aligned collagen fibrils compared to uninjured muscle, which is indicative of fibrotic scarring[37].Regenerated fiber diameter was significantly higher in animals treated with FTY720compared to PLGA only (Figure7C, D), and comparable to that observed qualitatively in uninjured spinotrapezius muscle. Taken together, these findings demonstrate that immunomodulatory biomaterial strategies that tune myeloid cell infiltration can improve skeletal muscle repair. 


\section{$\underline{\text { Discussion }}$}

In the present work, we have demonstrated that blood-derived non-classical monocytes infiltrate skeletal muscle after volumetric injury and convert into perivascular CD68+CD206+ macrophages. Biomaterial-based strategies that increase recruitment of non-classical monocytes concomitantly increase perivascular accumulation of AAMs, which correlates with the initiation of repair programs such as injury site re-vascularization, collagen deposition, and regeneration of damaged skeletal muscle. We demonstrate that accelerating the progression of muscle healing by targeting pro-regenerative monocytes and macrophage populations improves muscle regeneration while minimizing fibrotic scarring.

Volumetric muscle injury appears to elicit an inflammatory cascade similar to that observed in toxin-induced injury with peakaccumulation of Ly6C ${ }^{\text {hi }}$ monocytesat 1daypostmuscle injury and continued increase in Ly6C ${ }^{\text {lo }}$ monocytes following toxin insult [3]. Within the spinotrapezius VML model, Ly6C ${ }^{\text {hi }}$ inflammatory monocytes are present at 1 day post-injury and decrease over time, whereas Ly6C ${ }^{\text {lo }}$ anti-inflammatory monocytes peak at 3 days post-injury. Bothmonocyte subsets may exert pro-regenerative effects through productionof keyenzymatic and soluble cues such as inducible nitric oxide synthase (iNOS) by Ly6C ${ }^{\text {hi }}$ monocytes[38, 39], andvascular endothelial growth factor (VEGF)[40] and IGF-1[41] by Ly6C ${ }^{\text {lo }}$ monocytes. Once extravasated, however, monocytes mostly differentiate into macrophages that continue to support repair[42]. In vitro, monocyte/macrophage subpopulations exert differential effects on myogenic precursors through their secreted factors[8]. Conditioned media from inflammatory macrophagespromotes myogenic precursor cell proliferation and motility, whereas the soluble factors from anti-inflammatory macrophages promote myogenic differentiation and myotube formation[3, 8].Monocytes and macrophages may also direct their own behavior and fate through 
paracrine and autocrine signaling. Monocytes and macrophages produce tumor necrosis factoralpha (TNF- $\alpha$ ) and IL-1 $\beta$ which leads to self-amplification of inflammatory responses[43], and their phagocytosis of cellular debris results in downregulation of inflammatory cytokine expression[44].Thus, controlling the accumulation of specific monocyte and macrophage populations in vivorepresents an important step inbetter understanding their role in the intricate wound healing process.

Several studies have documented the recruitment of Ly6C ${ }^{\text {hi }}$ inflammatory monocytes to injury where they convert in situ to Ly6 $\mathrm{C}^{\mathrm{lo}}$ monocytes and become the primary contributor to wound macrophages[3, 45-47]. Conversely, accounts of direct recruitment of Ly6C ${ }^{\text {lo }}$ monocytes have been reported in myocardial infarction[40] and excisional skin injury[25]. Recruitment of non-classical Ly6 $\mathrm{C}^{\mathrm{lo}}$ monocytes may therefore be dependent on the type of injury and tissue of origin. Wehave built upon this knowledge by demonstrating for the first time that endogenous Ly6C $^{\text {lo }}$ monocytes are directly recruited to VML (Figure 3C), suggesting that Ly6C ${ }^{\text {lo }}$ monocytes may play an indispensable role in the healing of traumatic injuries that require the de novo growth of tissue.Use ofLX bead-based labeling of Ly6C ${ }^{\text {lo }}$ monocytes in the CX3CR1 $1^{\mathrm{GFP} /+}$ mouseenables tracking of GFP+LX+ monocytes in tissue, and also LX+ cells that may have lost GFP expression due to differentiation into macrophages[27]. LX+ cells appear in spinotrapezius muscle following VML (Figure 3C, D), indicating direct recruitment of $\mathrm{LyCC}^{\mathrm{lo}}$ monocytes from circulation.Important to the interpretation of trafficking studies, previous work has indicated that intravenous LX bead administration does not alter the kinetics of Ly6C ${ }^{\text {hi }}$ and $\mathrm{Ly}_{6 \mathrm{C}}{ }^{\mathrm{lo}}$ monocyte recruitment [27]. Additionally, LX bead administration does not induce changes in monocyte gene expression or systemically elevate plasma inflammatory mediators such as TNF- $\alpha$, IFN- $\gamma$, or IL-6[27]. In CX3CR1 ${ }^{\mathrm{GFP} /+}$ mice, blood monocyte compositionis the same compared to 
wildtype mice[48, 49]and CX3CR1 is not required for transendothelial migration of monocytes in a model of acute peritonitis[50], thus simplifying interpretation of data collected in CX3CR1 $1^{\mathrm{GFP} /+}$ mice.

VML defect healing follows similar phases to that of wound healing in injured skin [31]. 3D confocal microscopy ofexplanted spinotrapezius muscles at 3 days post-injury shows granulation tissue and nascent vasculature within the defect next to truncated muscle fibers (Figure 1C, F). This region of granulation tissue appeared to co-localize with CD206+ cells.The distribution of $\mathrm{CD}^{206+}$ cells is consistent with studiesthat indicateAAMsdirectly promotecollagen production in fibroblasts[16]and support vascularization of Matrigel subcutaneous implants[19]. In the resolving phases of inflammation, muscle macrophages upregulate genes for ECMconstruction and remodeling[10], and depletion of these macrophages severely impairs muscle regeneration[3].These findings motivated us to investigate whetherpreferentially increasingAAMs within injured skeletal muscle would enhance healing of volumetric muscle defects.

Biomaterial-based therapies such as ECM-derived scaffolds and macrophage-polarizing hydrogels[23] canincrease theratio of anti-inflammatory to inflammatorymacrophages[51, 52]and improve regenerative outcome, but the origin of the AAMs (whether resident or circulation-derived) remains unknown. Recent intriguing insights into the functional differences between resident AAMs and those derived from circulating monocytes have highlighted the importance of considering AAM origin when designing pro-regenerative biomaterials. Monocyte-derived AAMs have a unique gene expression profile compared to tissue resident AAMs, upregulating genes important during inflammationsuch as Socs2, IL-31ra, Ccl17, Ch25h, Jag2, and Ccl22[53].Monocyte-derived AAMs also specifically drive the differentiation of 
CD4+ Tcells to Foxp3+ regulatory Tcellsin a retinoic acid dependent manner[53]. Foxp3+ regulatory Tcells arecrucial to the healing of damaged skeletal muscle[54], and increasing their recruitment via IL-33 delivery to injured skeletal muscle in aged mice improves regeneration[55].Therefore, circulating monocytes likely possess an intrinsic capacity for promoting wound repair and should be strongly considered in the design of biomaterials that seek to leverage pro-regenerative inflammation. Previously, we have used local delivery of the small molecule FTY720 as a means to localize circulating pro-regenerative monocytes to sites of bone and skin injury to coordinate microvascular expansion and bone healing. In this work, we utilized on-site delivery of the smallmolecule FTY720 to explore the contributions of circulating non-classicalmonocytes within VML injury. The effects of FTY720 are pleiotropic, as all cells express some combination of S1P receptors[56]. When administered systemically, FTY720 induces lymphopenia and bradycardia [56];however, systemic effectsare avoided during the local delivery strategiesthat we have extensively investigated in previous work [25, 26, 57] and the current study.

FTY720 delivery from a polymer PLGA thin film was able to significantly increase the frequency of Ly6C $\mathrm{C}^{\mathrm{lo}}$ monocytes within injured muscle (Figure 4A). Adoptively-transferred nonclassical monocytes differentiate into CD206+ macrophages in injured skin[25].Therefore, we investigatedwhetherincreasing non-classical monocyte frequency would also result in increased numbers of CD206+ macrophages. Analysis of CD68+CD206+ macrophages in peri-defect tissue revealed increased numbers of this population in FTY720-treated animals (Figure 5F).Monocytes and macrophages are known to position themselves strategically by remodeling blood vessels[25, 36, 58], where the crosstalk between vasculature and immune cells can provide instructional cues for AAMdifferentiation[59]. During chronic arterial occlusion, CD163+ 
AAMsare clusteredin the perivascular space at sites of collateral growth in remodeling vasculature[60]. Here, spatial analysis reveals that CD68+CD206+ cells are positioned significantly closer to vesselswith FTY720 treatment(Figure 5D). This increased perivascular positioning of AAMs coincides with increased density of vasculature in the muscle defect area (Figure 6 A,B) compared to PLGA control animals. AAMsalso support healing of damaged skeletal muscle by providing a source of regenerative growth factors such as IGF-1[11]and promoting satellite cell differentiation into mature myotubes[3, 8]. AAMs control collagen deposition from fibroblasts in an IL-4ro-dependent manner[15], a process that is vital to wound healing within the skin. Assessment of the defect area via SHG imaging of collagen showed that FTY720 results in increased collagen content and a smallerdefect area 3 days post-injury (Figure 6C-F). By 7dayspost-injury, regenerated fiber diameter was significantly higher in FTY720 animals and the collagen architecture more closely mimicked that of native uninjured muscle (Figure 7C, D).

Though we have focused primarily on FTY720's effects on the behavior of monocytes and macrophages, agonism of S1PR1 and S1PR3 on other cell typespresent within the injury may have contributed to the accelerated healingwe observed in muscle regeneration. S1PR1 and S1PR3 aredifferentially expressedduring toxin-induced muscle injury, indicating that specific S1P receptors differentially regulate phases of healing[61]. S1PR3in muscle tissue is highly expressed during the acute inflammatory phase post-injury, and S1PR3 may positively regulate the growth of regenerating fibers[61]. Conversely, S1PR1 expression increases during regeneration and S1PR1 agonism produced a net decrease in regenerated fiber cross-sectional area[61].Geneticknockout of S1PR3 promotessatellite cellproliferation and attenuates muscle degeneration in the mdx mouse model that simulates Duchenne muscular dystrophy[62]. Our 
work demonstrates that the signaling induced by FTY720 likely affects multiple cell types during muscle repair, given that we observed changes in myeloid cells, blood vessels, and myofibers. Because the current work is unable to distinguish between direct and paracrine effects induced by FTY720, additional studies employing loss-of-function models are needed to understand the precise interplay between different compartments during skeletal muscle repair.

A major clinical concern with large volumetric muscle injuries is the debilitating fibrosis that often occurs in place of satisfactory healing[18, 63].Fibrotic muscle not only lacks the contractilityof healthy muscle, but also exhibits decreased elasticity, rendering the tissue more susceptible to re-injury[64]. Whilegranulation tissue formation and its subsequent remodeling into mature ECM is crucial for the restoration of functional tissue in the volumetric void, a fibrotic response often progresses faster than a myogenic response[65].Macrophages have the capacity to sense the injury microenvironment and instruct fibroblasts to either continue producing matrix or undergo apoptosis[17]. Increasing the frequency of AAMs may provide an endogenous command centercapable of controlling and alleviatinghyper-fibrotic responses within volumetric muscle defects. We utilized 3D analysis of the SHG collagen signal along with fluorescently-labeled desmin to determine extent of fibrosis between fibers 7days post-injury. Strikingly, areas of regrown fibers within FTY720-treated animals displayed a lower ratio of collagen to desmin volume (Figure 7B), indicating either faster healing in place of fibrosis, or attenuation of hyper-fibrotic responses in the defect to allow for increased parenchymal muscle fiber ingrowth. These observations are particularly promisingfor the treatment of larger, critically sized volumetric defects where prolonged fibrotic response after injury results in impaired progenitor and stromal cell migration and subsequently, decreased angiogenesis, reinnervation, and skeletal muscle regrowth[66, 67]. Future studies should explore how acute modulation of 
inflammatory cell recruitment impacts long-term reconstruction of critical sized traumatic muscle injury.Taken together, our data strongly suggest that greater accumulation of CD68+CD206+ cells achieved through increased recruitment of non-classical Ly6C ${ }^{\text {lo }}$ monocytespromotes early volumetric muscle wound closure and improves healing outcome within regenerated muscle. These findings indicate that localizing endogenous pro-regenerative inflammation with material-based biomolecule delivery is a promising strategy to promote healing of traumatic musculoskeletal injuries. 


\section{Acknowledgements}

We thank the core facilities staff of the Parker H. Petit Institute for Bioengineering and Bioscience for technical expertise and assistance (particularly Andrew Shaw and Nadia Bugoslavsky), the Physiological Research Lab staff for guidance on in vivo studies, and the Emory University Integrated Cellular Imaging Microscopy Core for their expertise and assistance in image analysis.

\section{Sources of Funding}

This work was supported by the National Institutes of Health grants, R01AR056445, and R01DE019935 to Dr. Botchwey, National Science Foundation Graduate Research Fellowship under Grant No. DGE-1148903, American Heart Association Grant 15PRE25090024 (Claire Segar), and P.E.O. Scholar Award to Claire Segar.

\section{Animal Experiment Compliance Statement}

All animal experiments were conducted in accordance with ARRIVE guidelines and the National Institutes of Health guide for the Care and Use of Laboratory Animals. 


\section{$\underline{\text { References }}$}

[1] H. Yin, F. Price, M.A. Rudnicki, Satellite Cells and the Muscle Stem Cell Niche, Physiological Reviews 93(1) (2013) 23-67.

[2] H. Wang, D.W. Melton, L. Porter, Z.U. Sarwar, L.M. McManus, P.K. Shireman, Altered macrophage phenotype transition impairs skeletal muscle regeneration, The American journal of pathology 184(4) (2014) 1167-84.

[3] L. Arnold, A. Henry, F. Poron, Y. Baba-Amer, N. van Rooijen, A. Plonquet, R.K. Gherardi, B. Chazaud, Inflammatory monocytes recruited after skeletal muscle injury switch into antiinflammatory macrophages to support myogenesis, The Journal of experimental medicine 204(5) (2007) 1057-69.

[4] M. Summan, G.L. Warren, R.R. Mercer, R. Chapman, T. Hulderman, N. Van Rooijen, P.P. Simeonova, Macrophages and skeletal muscle regeneration: a clodronate-containing liposome depletion study, American Journal of Physiology - Regulatory, Integrative and Comparative Physiology 290(6) (2006) R1488-R1495.

[5] S. Willenborg, T. Lucas, G. van Loo, J.A. Knipper, T. Krieg, I. Haase, B. Brachvogel, M. Hammerschmidt, A. Nagy, N. Ferrara, M. Pasparakis, S.A. Eming, CCR2 recruits an inflammatory macrophage subpopulation critical for angiogenesis in tissue repair, Blood 120(3) (2012) 613-25.

[6] D. Dal-Secco, J. Wang, Z. Zeng, E. Kolaczkowska, C.H. Wong, B. Petri, R.M. Ransohoff, I.F. Charo, C.N. Jenne, P. Kubes, A dynamic spectrum of monocytes arising from the in situ reprogramming of CCR2+ monocytes at a site of sterile injury, The Journal of experimental medicine 212(4) (2015) 447-56. 
[7] I. Avraham-Davidi, S. Yona, M. Grunewald, L. Landsman, C. Cochain, J.S. Silvestre, H. Mizrahi, M. Faroja, D. Strauss-Ayali, M. Mack, S. Jung, E. Keshet, On-site education of VEGFrecruited monocytes improves their performance as angiogenic and arteriogenic accessory cells, The Journal of experimental medicine 210(12) (2013) 2611-25.

[8] M. Saclier, H. Yacoub-Youssef, A.L. Mackey, L. Arnold, H. Ardjoune, M. Magnan, F. Sailhan, J. Chelly, G.K. Pavlath, R. Mounier, M. Kjaer, B. Chazaud, Differentially activated macrophages orchestrate myogenic precursor cell fate during human skeletal muscle regeneration, Stem Cells 31(2) (2013) 384-96.

[9] M.J. Crane, J.M. Daley, O. van Houtte, S.K. Brancato, W.L. Henry, Jr., J.E. Albina, The monocyte to macrophage transition in the murine sterile wound, PloS one 9(1) (2014) e86660.

[10] T. Varga, R. Mounier, A. Horvath, S. Cuvellier, F. Dumont, S. Poliska, H. Ardjoune, G. Juban, L. Nagy, B. Chazaud, Highly Dynamic Transcriptional Signature of Distinct Macrophage Subsets during Sterile Inflammation, Resolution, and Tissue Repair, The Journal of Immunology 96(11) (2016) 4771-82.

[11] J. Tonkin, L. Temmerman, R.D. Sampson, E. Gallego-Colon, L. Barberi, D. Bilbao, M.D. Schneider, A. Musaro, N. Rosenthal, Monocyte/Macrophage-derived IGF-1 Orchestrates Murine Skeletal Muscle Regeneration and Modulates Autocrine Polarization, Mol Ther 23(7) (2015) 1189-200.

[12] F. Ginhoux, M. Greter, M. Leboeuf, S. Nandi, P. See, S. Gokhan, M.F. Mehler, S.J. Conway, L.G. Ng, E.R. Stanley, I.M. Samokhvalov, M. Merad, Fate mapping analysis reveals that adult microglia derive from primitive macrophages, Science (New York, N.Y.) 330(6005) (2010) 841-5. 
[13] S. Yona, K.W. Kim, Y. Wolf, A. Mildner, D. Varol, M. Breker, D. Strauss-Ayali, S. Viukov, M. Guilliams, A. Misharin, D.A. Hume, H. Perlman, B. Malissen, E. Zelzer, S. Jung, Fate mapping reveals origins and dynamics of monocytes and tissue macrophages under homeostasis, Immunity 38(1) (2013) 79-91.

[14] J. Sottile, Regulation of angiogenesis by extracellular matrix, Biochimica et biophysica acta 1654(1) (2004) 13-22.

[15] J.A. Knipper, S. Willenborg, J. Brinckmann, W. Bloch, T. Maass, R. Wagener, T. Krieg, T. Sutherland, A. Munitz, M.E. Rothenberg, A. Niehoff, R. Richardson, M. Hammerschmidt, J.E. Allen, S.A. Eming, Interleukin-4 Receptor alpha Signaling in Myeloid Cells Controls Collagen Fibril Assembly in Skin Repair, Immunity 43(4) (2015) 803-16.

[16] E. Song, N. Ouyang, M. Horbelt, B. Antus, M. Wang, M.S. Exton, Influence of alternatively and classically activated macrophages on fibrogenic activities of human fibroblasts, Cellular immunology 204(1) (2000) 19-28.

[17] D.R. Lemos, F. Babaeijandaghi, M. Low, C.K. Chang, S.T. Lee, D. Fiore, R.H. Zhang, A. Natarajan, S.A. Nedospasov, F.M. Rossi, Nilotinib reduces muscle fibrosis in chronic muscle injury by promoting TNF-mediated apoptosis of fibro/adipogenic progenitors, Nature medicine 21(7) (2015) 786-94.

[18] K. Garg, C.L. Ward, B.J. Hurtgen, J.M. Wilken, D.J. Stinner, J.C. Wenke, J.G. Owens, B.T. Corona, Volumetric muscle loss: Persistent functional deficits beyond frank loss of tissue, Journal of Orthopaedic Research 33(1) (2015) 40-46.

[19] N. Jetten, S. Verbruggen, M.J. Gijbels, M.J. Post, M.P. De Winther, M.M. Donners, Antiinflammatory M2, but not pro-inflammatory M1 macrophages promote angiogenesis in vivo, Angiogenesis 17(1) (2014) 109-18. 
[20] K.L. Spiller, R.R. Anfang, K.J. Spiller, J. Ng, K.R. Nakazawa, J.W. Daulton, G. VunjakNovakovic, The role of macrophage phenotype in vascularization of tissue engineering scaffolds, Biomaterials 35(15) (2014) 4477-88.

[21] Y. Takeda, S. Costa, E. Delamarre, C. Roncal, R. Leite de Oliveira, M.L. Squadrito, V. Finisguerra, S. Deschoemaeker, F. Bruyere, M. Wenes, A. Hamm, J. Serneels, J. Magat, T. Bhattacharyya, A. Anisimov, B.F. Jordan, K. Alitalo, P. Maxwell, B. Gallez, Z.W. Zhuang, Y. Saito, M. Simons, M. De Palma, M. Mazzone, Macrophage skewing by Phd2 haplodeficiency prevents ischaemia by inducing arteriogenesis, Nature 479(7371) (2011) 122-126.

[22] T.A.H. Järvinen, T.L.N. Järvinen, M. Kääriäinen, H. Kalimo, M. Järvinen, Muscle Injuries: Biology and Treatment, The American Journal of Sports Medicine 33(5) (2005) 745-764.

[23] N. Mokarram, A. Merchant, V. Mukhatyar, G. Patel, R.V. Bellamkonda, Effect of modulating macrophage phenotype on peripheral nerve repair, Biomaterials 33(34) (2012) 87938801.

[24] J. Rivera, R.L. Proia, A. Olivera, THE ALLIANCE OF SPHINGOSINE-1-PHOSPHATE AND ITS RECEPTORS IN IMMUNITY, Nature reviews. Immunology 8(10) (2008) 753-763.

[25] A.O. Awojoodu, M.E. Ogle, L.S. Sefcik, D.T. Bowers, K. Martin, K.L. Brayman, K.R. Lynch, S.M. Peirce-Cottler, E. Botchwey, Sphingosine 1-phosphate receptor 3 regulates recruitment of anti-inflammatory monocytes to microvessels during implant arteriogenesis, Proceedings of the National Academy of Sciences 110 (34) (2013) 13785-90.

[26] A. Das, C.E. Segar, B.B. Hughley, D.T. Bowers, E.A. Botchwey, The promotion of mandibular defect healing by the targeting of S1P receptors and the recruitment of alternatively activated macrophages, Biomaterials 34(38) (2013) 9853-62. 
[27] F. Tacke, D. Alvarez, T.J. Kaplan, C. Jakubzick, R. Spanbroek, J. Llodra, A. Garin, J. Liu, M. Mack, N. van Rooijen, S.A. Lira, A.J. Habenicht, G.J. Randolph, Monocyte subsets differentially employ CCR2, CCR5, and CX3CR1 to accumulate within atherosclerotic plaques, J Clin Invest 117(1) (2007) 185-94.

[28] A.M. Guendel, K.S. Martin, J. Cutts, P.L. Foley, A.M. Bailey, F. Mac Gabhann, T.R. Cardinal, S.M. Peirce, Murine spinotrapezius model to assess the impact of arteriolar ligation on microvascular function and remodeling, Journal of visualized experiments : JoVE (73) (2013) e50218.

[29] A.M. Bailey, T.J.t. O'Neill, C.E. Morris, S.M. Peirce, Arteriolar remodeling following ischemic injury extends from capillary to large arteriole in the microcirculation, Microcirculation (New York, N.Y. : 1994) 15(5) (2008) 389-404.

[30] L. Micallef, N. Vedrenne, F. Billet, B. Coulomb, I.A. Darby, A. Desmoulière, The myofibroblast, multiple origins for major roles in normal and pathological tissue repair, Fibrogenesis \& Tissue Repair 5(1) (2012) 1-5.

[31] G.C. Gurtner, S. Werner, Y. Barrandon, M.T. Longaker, Wound repair and regeneration, Nature 453 (7193) (2008) 314-21.

[32] E.L. Gautier, T. Shay, J. Miller, M. Greter, C. Jakubzick, S. Ivanov, J. Helft, A. Chow, K.G. Elpek, S. Gordonov, A.R. Mazloom, A. Ma'ayan, W.J. Chua, T.H. Hansen, S.J. Turley, M. Merad, G.J. Randolph, Gene-expression profiles and transcriptional regulatory pathways that underlie the identity and diversity of mouse tissue macrophages, Nature immunology 13(11) (2012) 1118-28. 
[33] D. Pilling, T. Fan, D. Huang, B. Kaul, R.H. Gomer, Identification of Markers that Distinguish Monocyte-Derived Fibrocytes from Monocytes, Macrophages, and Fibroblasts, PloS one 4(10) (2009) e7475.

[34] A. Wollenberg, M. Mommaas, T. Oppel, E.M. Schottdorf, S. Gunther, M. Moderer, Expression and function of the mannose receptor CD206 on epidermal dendritic cells in inflammatory skin diseases, J Invest Dermatol 118(2) (2002) 327-34.

[35] E. Zigmond, C. Varol, J. Farache, E. Elmaliah, A.T. Satpathy, G. Friedlander, M. Mack, N. Shpigel, I.G. Boneca, K.M. Murphy, G. Shakhar, Z. Halpern, S. Jung, Ly6C hi monocytes in the inflamed colon give rise to proinflammatory effector cells and migratory antigen-presenting cells, Immunity 37(6) (2012) 1076-90.

[36] A.C. Bruce, M.R. Kelly-Goss, J.L. Heuslein, J.K. Meisner, R.J. Price, S.M. Peirce, Monocytes are recruited from venules during arteriogenesis in the murine spinotrapezius ligation model, Arteriosclerosis, thrombosis, and vascular biology 34(9) (2014) 2012-22.

[37] S. McDougall, J. Dallon, J. Sherratt, P. Maini, Fibroblast migration and collagen deposition during dermal wound healing: mathematical modelling and clinical implications, Philosophical transactions. Series A, Mathematical, physical, and engineering sciences 364(1843) (2006) 1385405.

[38] E. Rigamonti, T. Touvier, E. Clementi, A.A. Manfredi, S. Brunelli, P. Rovere-Querini, Requirement of Inducible Nitric Oxide Synthase for Skeletal Muscle Regeneration after Acute Damage, J Immunol 190(4) (2013) 1767-77.

[39] I.R. Dunay, R.A. Damatta, B. Fux, R. Presti, S. Greco, M. Colonna, L.D. Sibley, Gr1(+) inflammatory monocytes are required for mucosal resistance to the pathogen Toxoplasma gondii, Immunity 29(2) (2008) 306-17. 
[40] M. Nahrendorf, F.K. Swirski, E. Aikawa, L. Stangenberg, T. Wurdinger, J.L. Figueiredo, P. Libby, R. Weissleder, M.J. Pittet, The healing myocardium sequentially mobilizes two monocyte subsets with divergent and complementary functions, J Exp Med 204(12) (2007) 3037-47.

[41] S.L. Lin, A.P. Castano, B.T. Nowlin, M.L. Lupher, Jr., J.S. Duffield, Bone marrow Ly6Chigh monocytes are selectively recruited to injured kidney and differentiate into functionally distinct populations, J Immunol 183(10) (2009) 6733-43.

[42] S. Gordon, P.R. Taylor, Monocyte and macrophage heterogeneity, Nat Rev Immunol 5(12) (2005) 953-64.

[43] J. Kajahn, S. Franz, E. Rueckert, I. Forstreuter, V. Hintze, S. Moeller, J.C. Simon, Artificial extracellular matrices composed of collagen I and high sulfated hyaluronan modulate monocyte to macrophage differentiation under conditions of sterile inflammation, Biomatter 2(4) (2012) 226-73.

[44] V.A. Fadok, D.L. Bratton, A. Konowal, P.W. Freed, J.Y. Westcott, P.M. Henson, Macrophages that have ingested apoptotic cells in vitro inhibit proinflammatory cytokine production through autocrine/paracrine mechanisms involving TGF-beta, PGE2, and PAF, The Journal of clinical investigation 101(4) (1998) 890-8.

[45] N.M. Girgis, U.M. Gundra, L.N. Ward, M. Cabrera, U. Frevert, P. Loke, Ly6C(high) monocytes become alternatively activated macrophages in schistosome granulomas with help from CD4+ cells, PLoS pathogens 10(6) (2014) e1004080.

[46] L. Denney, W.L. Kok, S.L. Cole, S. Sanderson, A.J. McMichael, L.P. Ho, Activation of invariant NKT cells in early phase of experimental autoimmune encephalomyelitis results in differentiation of Ly6Chi inflammatory monocyte to M2 macrophages and improved outcome, J Immunol 189(2) (2012) 551-7. 
[47] P. Ramachandran, A. Pellicoro, M.A. Vernon, L. Boulter, R.L. Aucott, A. Ali, S.N. Hartland, V.K. Snowdon, A. Cappon, T.T. Gordon-Walker, M.J. Williams, D.R. Dunbar, J.R. Manning, N. van Rooijen, J.A. Fallowfield, S.J. Forbes, J.P. Iredale, Differential Ly-6C expression identifies the recruited macrophage phenotype, which orchestrates the regression of murine liver fibrosis, Proc Natl Acad Sci U S A 109(46) (2012) E3186-95.

[48] C. Auffray, D.K. Fogg, E. Narni-Mancinelli, B. Senechal, C. Trouillet, N. Saederup, J. Leemput, K. Bigot, L. Campisi, M. Abitbol, T. Molina, I. Charo, D.A. Hume, A. Cumano, G. Lauvau, F. Geissmann, CX(3)CR1(+) CD115(+) CD135(+) common macrophage/DC precursors and the role of $\mathrm{CX}(3) \mathrm{CR} 1$ in their response to inflammation, The Journal of experimental medicine 206(3) (2009) 595-606.

[49] L. Landsman, L. Bar-On, A. Zernecke, K.W. Kim, R. Krauthgamer, E. Shagdarsuren, S.A. Lira, I.L. Weissman, C. Weber, S. Jung, CX3CR1 is required for monocyte homeostasis and atherogenesis by promoting cell survival, Blood 113(4) (2009) 963-72.

[50] S. Jung, J. Aliberti, P. Graemmel, M.J. Sunshine, G.W. Kreutzberg, A. Sher, D.R. Littman, Analysis of Fractalkine Receptor CX(3)CR1 Function by Targeted Deletion and Green Fluorescent Protein Reporter Gene Insertion, Molecular and Cellular Biology 20(11) (2000) 4106-14.

[51] B.N. Brown, J.E. Valentin, A.M. Stewart-Akers, G.P. McCabe, S.F. Badylak, Macrophage phenotype and remodeling outcomes in response to biologic scaffolds with and without a cellular component, Biomaterials 30(8) (2009) 1482-91.

[52] S.F. Badylak, J.E. Valentin, A.K. Ravindra, G.P. McCabe, A.M. Stewart-Akers, Macrophage phenotype as a determinant of biologic scaffold remodeling, Tissue engineering. Part A 14(11) (2008) 1835-42. 
[53] U.M. Gundra, N.M. Girgis, D. Ruckerl, S. Jenkins, L.N. Ward, Z.D. Kurtz, K.E. Wiens, M.S. Tang, U. Basu-Roy, A. Mansukhani, J.E. Allen, P. Loke, Alternatively activated macrophages derived from monocytes and tissue macrophages are phenotypically and functionally distinct, Blood 123(20) (2014) e110-22.

[54] D. Burzyn, W. Kuswanto, D. Kolodin, J.L. Shadrach, M. Cerletti, Y. Jang, E. Sefik, T.G. Tan, A.J. Wagers, C. Benoist, D. Mathis, A special population of regulatory T cells potentiates muscle repair, Cell 155(6) (2013) 1282-95.

[55] W. Kuswanto, D. Burzyn, M. Panduro, K.K. Wang, Y.C. Jang, A.J. Wagers, C. Benoist, D. Mathis, Poor Repair of Skeletal Muscle in Aging Mice Reflects a Defect in Local, Interleukin33-Dependent Accumulation of Regulatory T Cells, Immunity 44(2) (2016) 355-67.

[56] V.A. Blaho, T. Hla, An update on the biology of sphingosine 1-phosphate receptors, Journal of Lipid Research 55(8) (2014) 1596-608.

[57] M.E. Ogle, L.S. Sefcik, A.O. Awojoodu, N.F. Chiappa, K. Lynch, S. Peirce-Cottler, E.A. Botchwey, Engineering in vivo gradients of sphingosine-1-phosphate receptor ligands for localized microvascular remodeling and inflammatory cell positioning, Acta biomaterialia 10(11) (2014) 4704-14.

[58] A. Fantin, J.M. Vieira, G. Gestri, L. Denti, Q. Schwarz, S. Prykhozhij, F. Peri, S.W. Wilson, C. Ruhrberg, Tissue macrophages act as cellular chaperones for vascular anastomosis downstream of VEGF-mediated endothelial tip cell induction, Blood 116(5) (2010) 829-40.

[59] H. He, J. Xu, C.M. Warren, D. Duan, X. Li, L. Wu, M.L. Iruela-Arispe, Endothelial cells provide an instructive niche for the differentiation and functional polarization of M2-like macrophages, Blood 120(15) (2012) 3152-62. 
[60] C. Troidl, G. Jung, K. Troidl, J. Hoffmann, H. Mollmann, H. Nef, W. Schaper, C.W. Hamm, T. Schmitz-Rixen, The temporal and spatial distribution of macrophage subpopulations during arteriogenesis, Current vascular pharmacology 11(1) (2013) 5-12.

[61] D. Danieli-Betto, S. Peron, E. Germinario, M. Zanin, G. Sorci, S. Franzoso, D. Sandonà, R. Betto, Sphingosine 1-phosphate signaling is involved in skeletal muscle regeneration, American Journal of Physiology - Cell Physiology 298(3) (2010) C550-C558.

[62] M. Fortier, N. Figeac, R.B. White, P. Knopp, P.S. Zammit, Sphingosine-1-phosphate receptor 3 influences cell cycle progression in muscle satellite cells, Developmental Biology 382(2) (2013) 504-516.

[63] X. Wu, B.T. Corona, X. Chen, T.J. Walters, A standardized rat model of volumetric muscle loss injury for the development of tissue engineering therapies, BioResearch open access 1(6) (2012) 280-90.

[64] J. Huard, Y. Li, F.H. Fu, Muscle Injuries and Repair: Current Trends in Research, The Journal of Bone \& Joint Surgery 84(5) (2002) 822-832.

[65] K. Garg, B.T. Corona, T.J. Walters, Therapeutic strategies for preventing skeletal muscle fibrosis after injury, Frontiers in pharmacology 6 (2015) 87.

[66] B.T. Corona, K. Garg, C.L. Ward, J.S. McDaniel, T.J. Walters, C.R. Rathbone, Autologous minced muscle grafts: a tissue engineering therapy for the volumetric loss of skeletal muscle, American Journal of Physiology - Cell Physiology 305(7) (2013) C761-C775.

[67] B.M. Sicari, V. Agrawal, B.F. Siu, C.J. Medberry, C.L. Dearth, N.J. Turner, S.F. Badylak, A murine model of volumetric muscle loss and a regenerative medicine approach for tissue replacement, Tissue engineering. Part A 18(19-20) (2012) 1941-8. 


\section{$\underline{\text { Figures }}$}

Figure 1

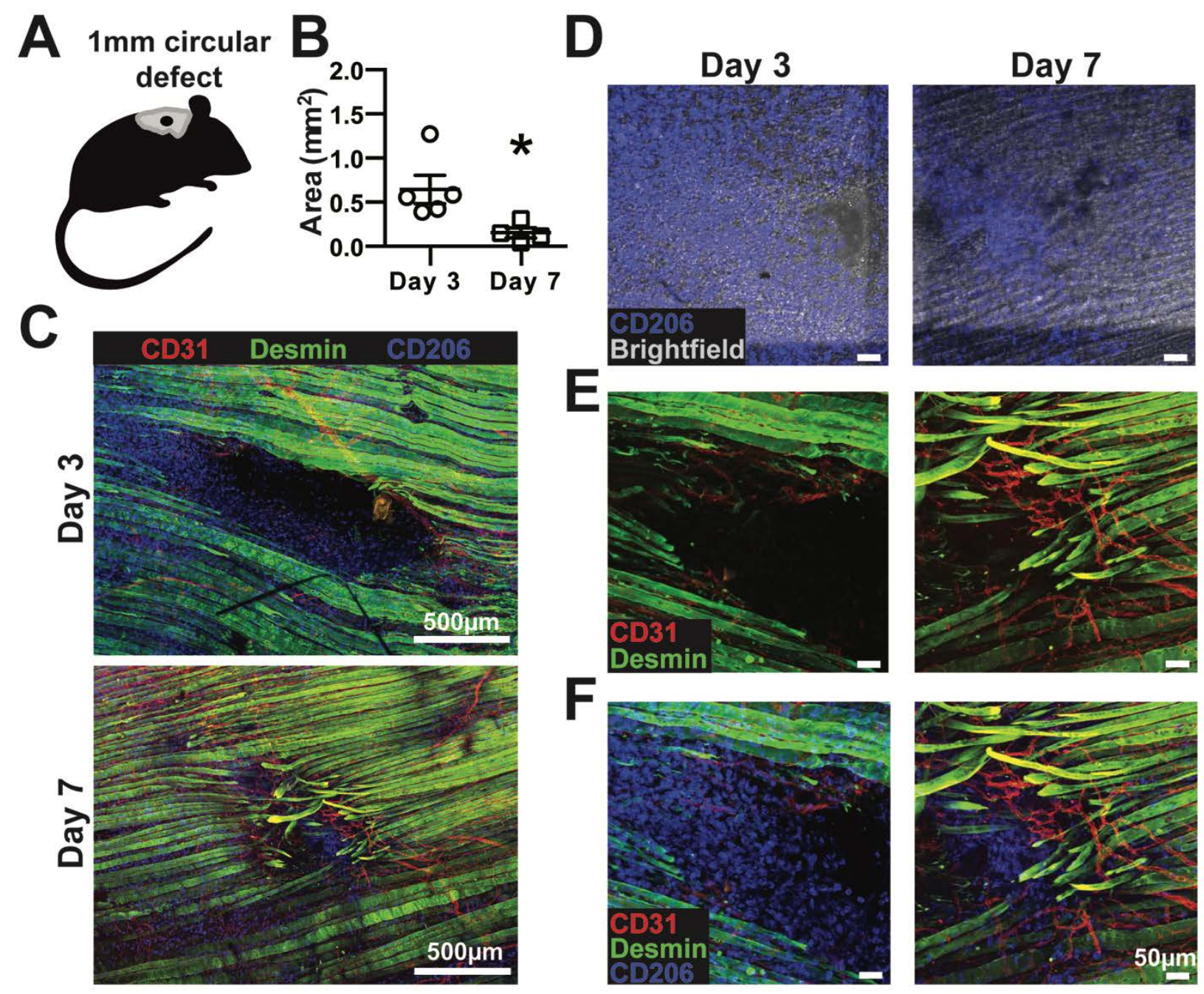


Figure 2
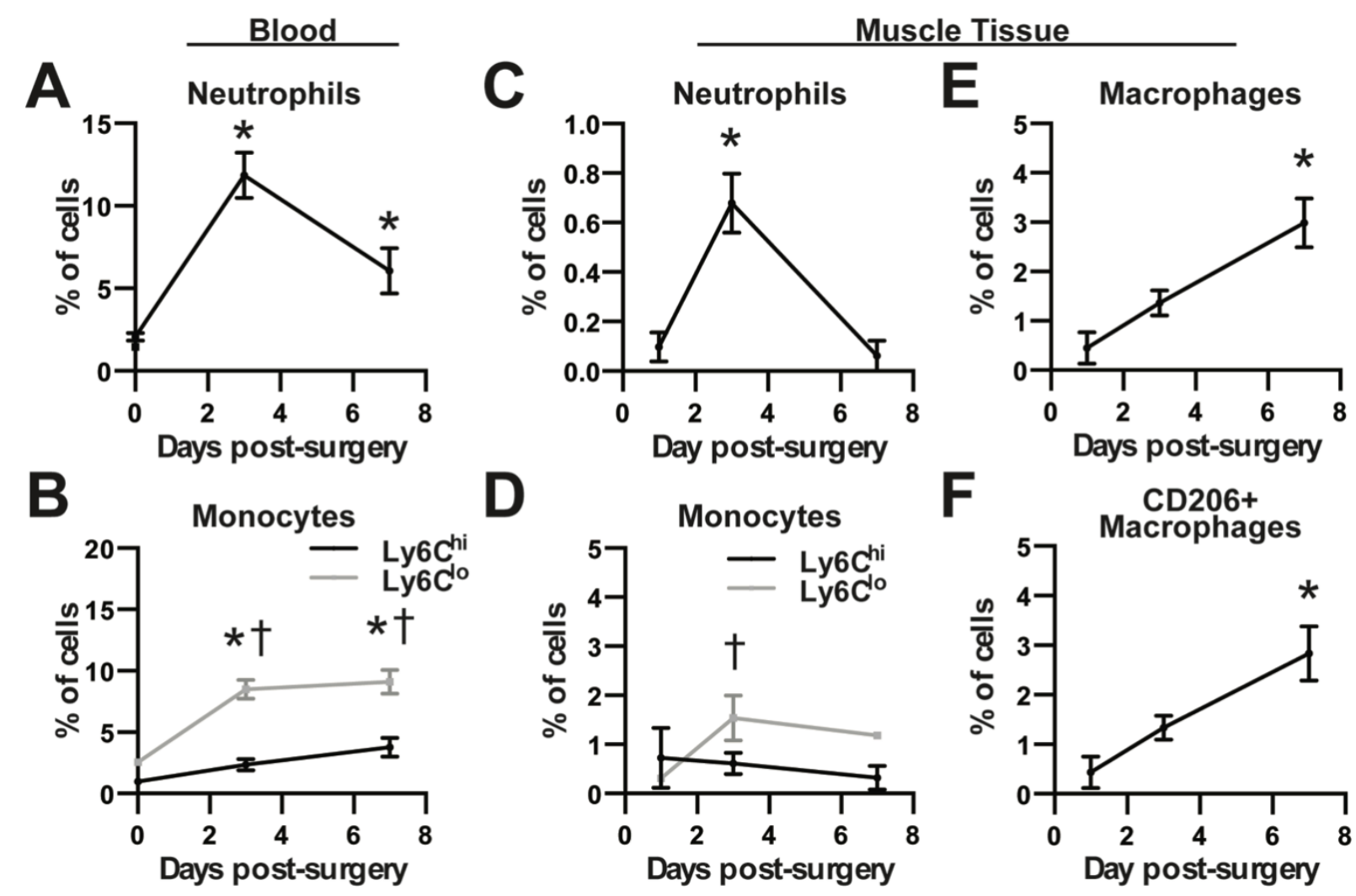
Figure 3

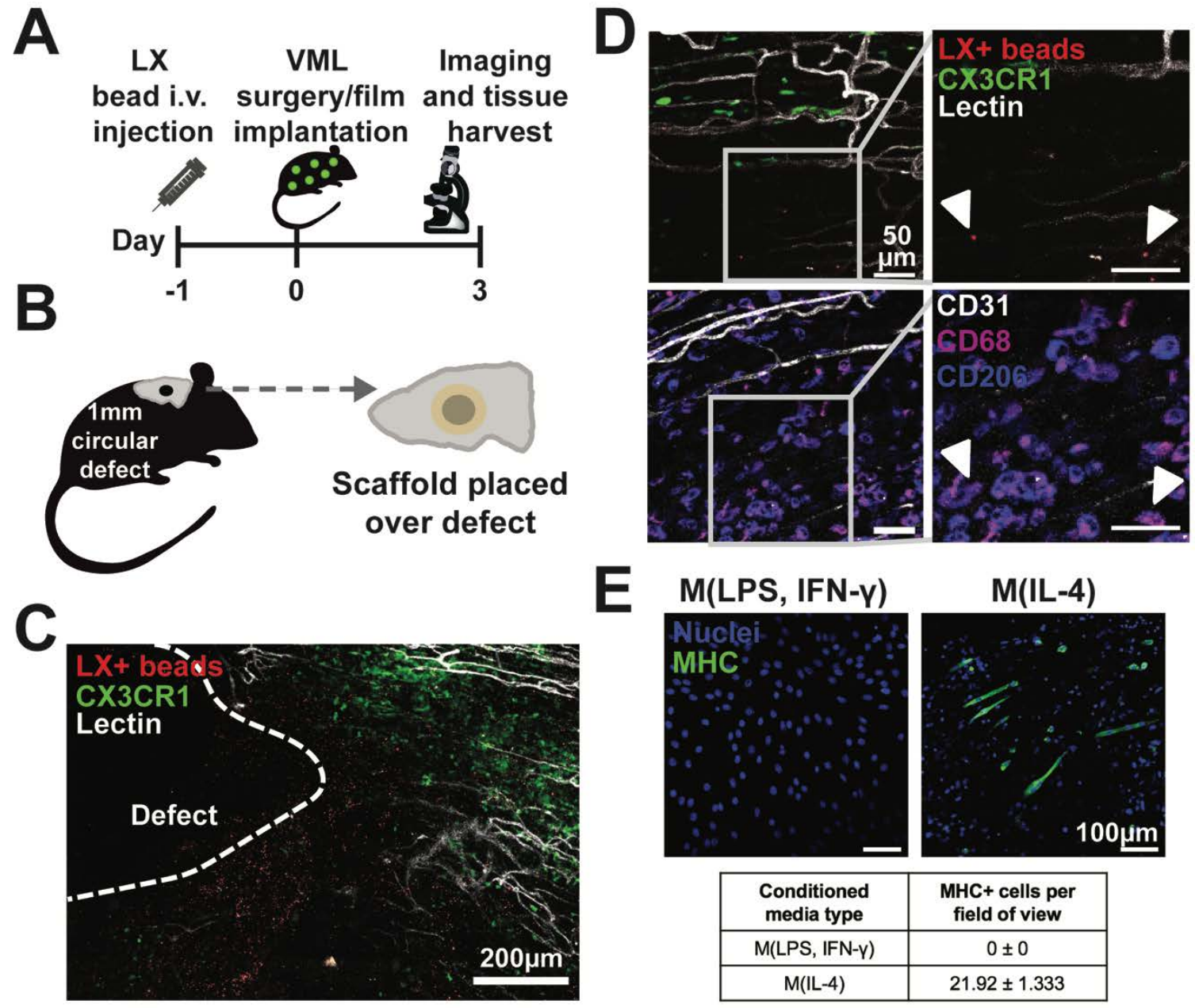


Figure 4

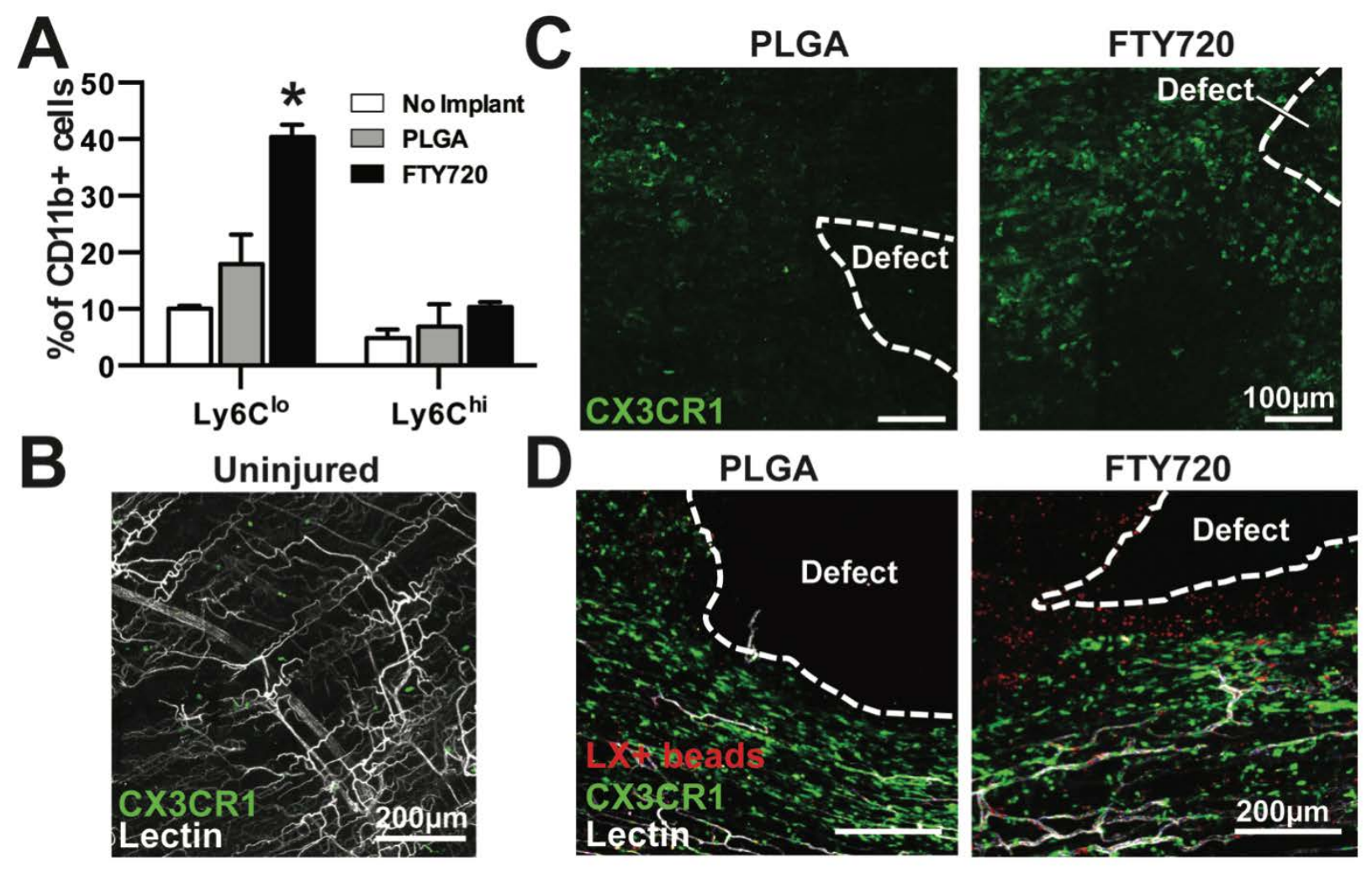


Figure 5
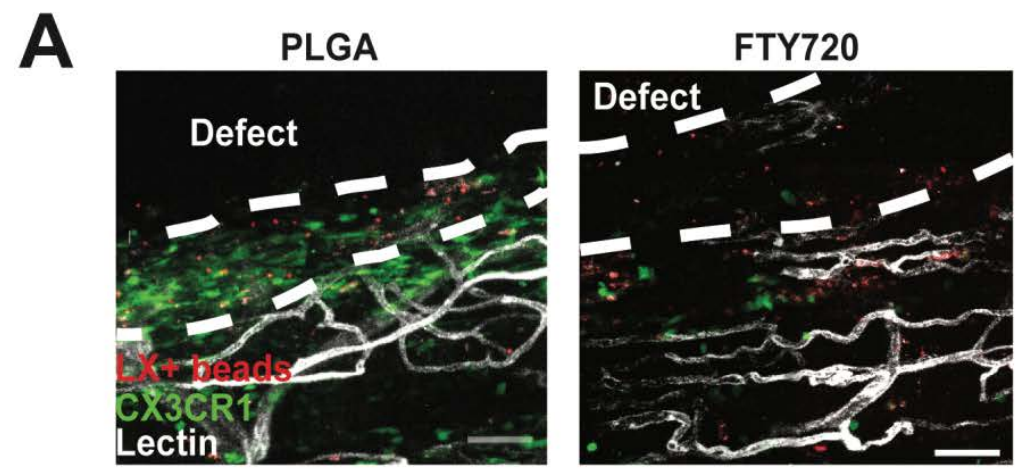

B

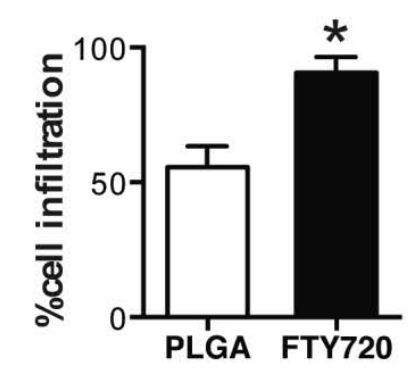

C
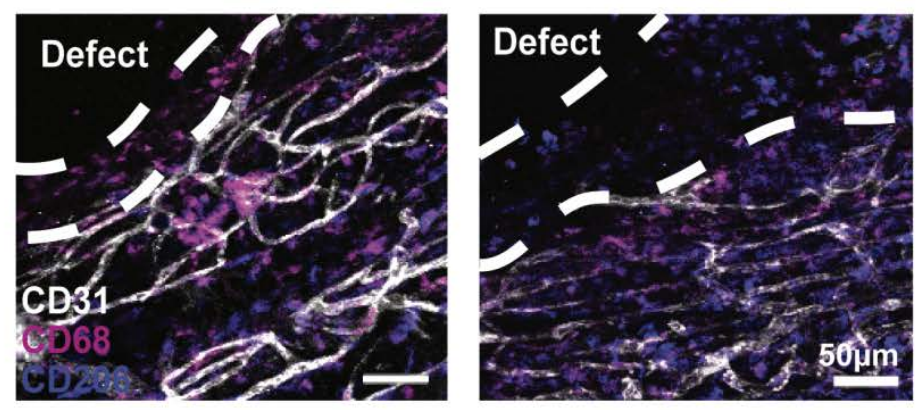

D
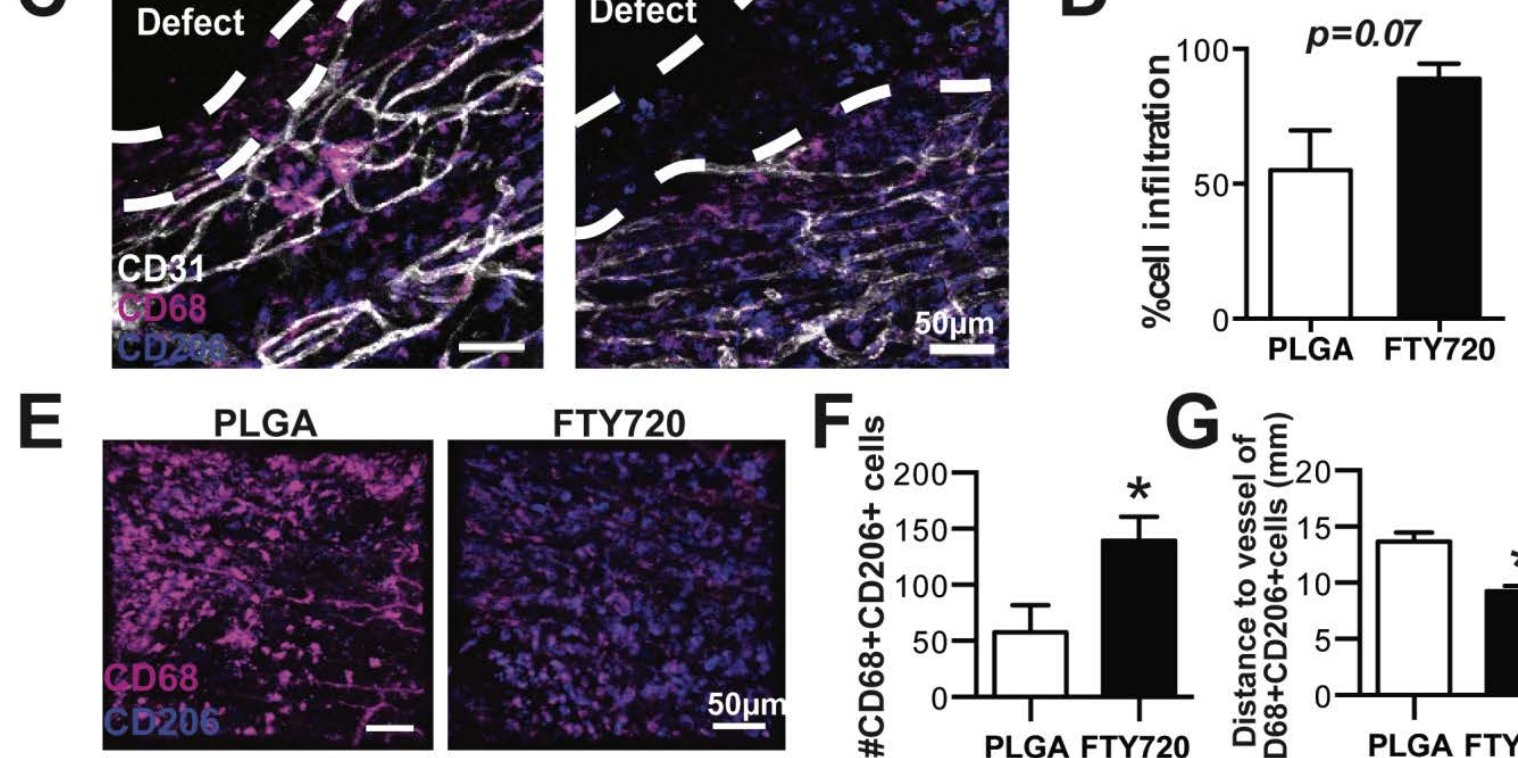

FTY720
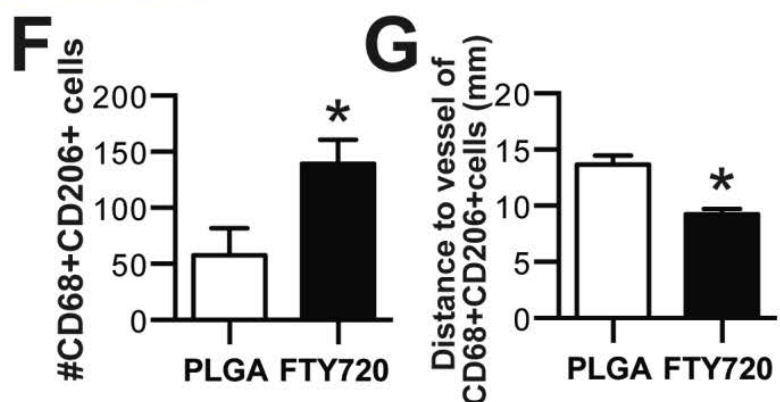
Figure 6

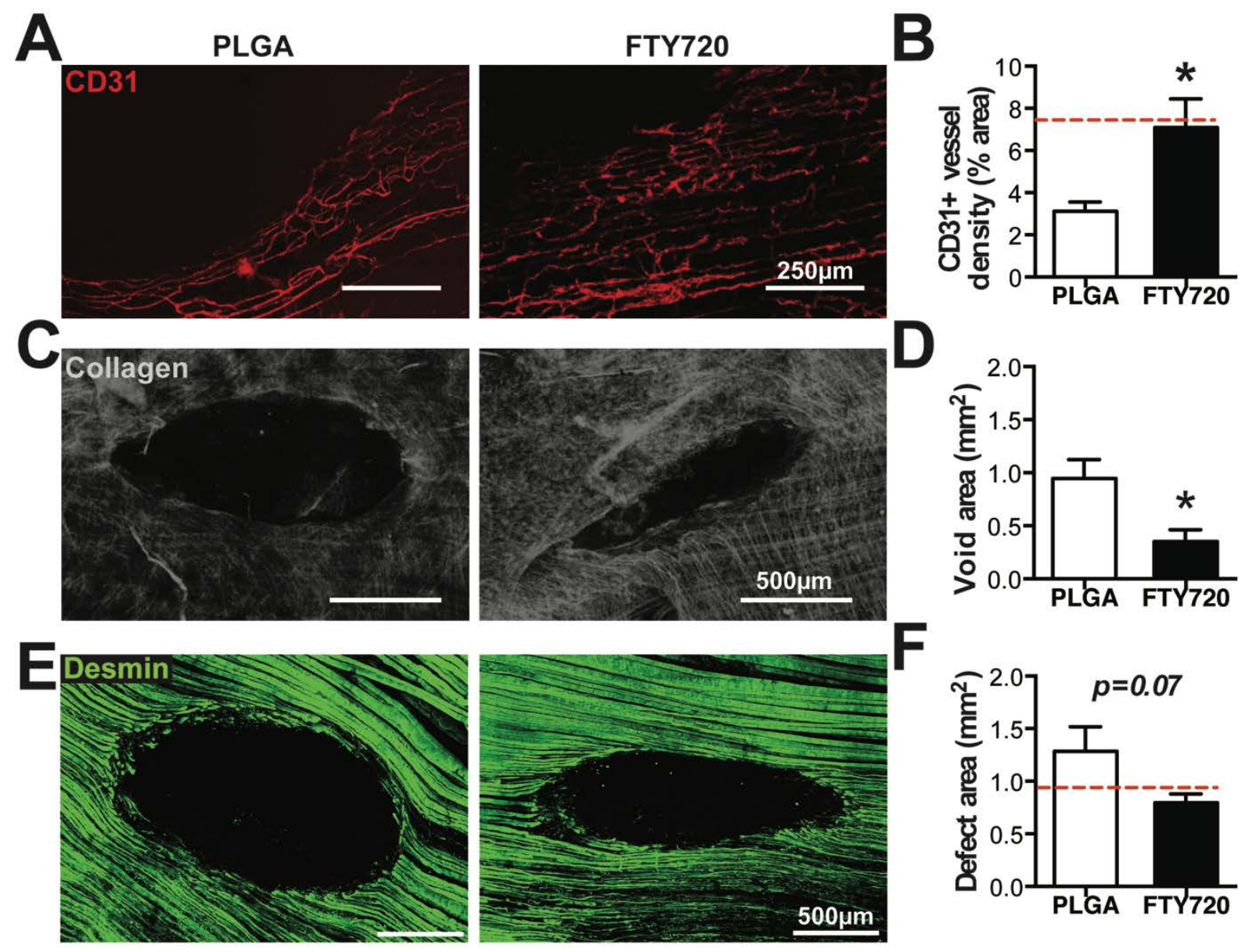


Figure 7

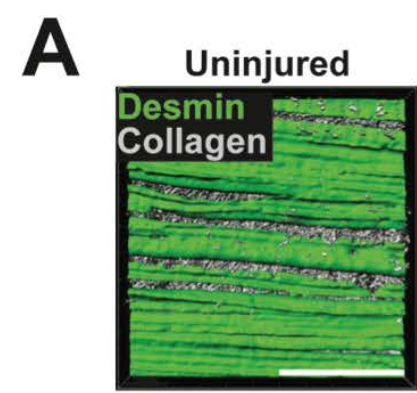

C

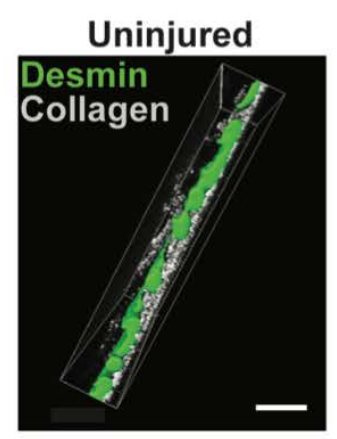

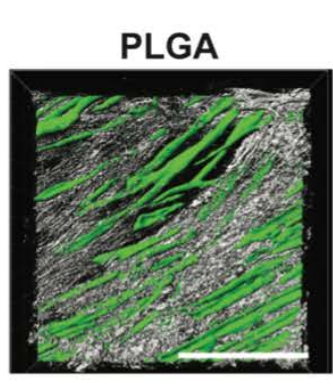

PLGA

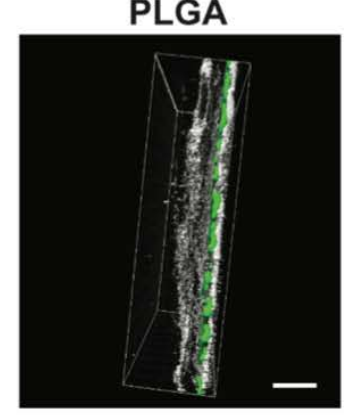

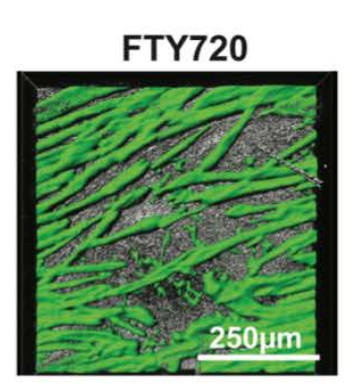

FTY720

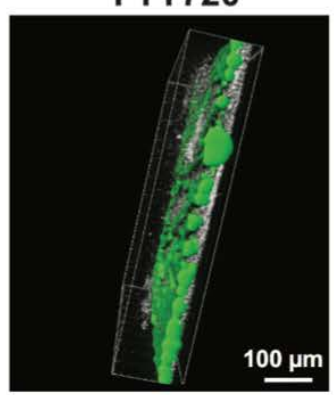

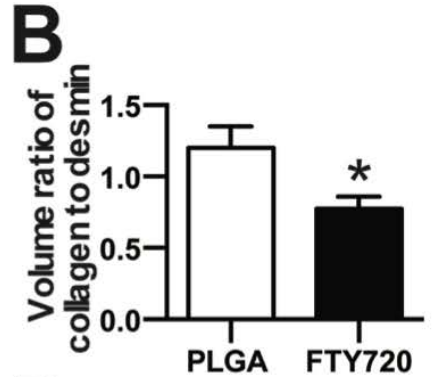

D

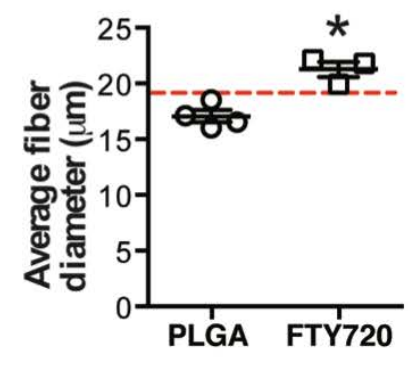

\section{$\underline{\text { Figure Captions }}$}

Figure 1. A novel volumetric muscle loss model in the murine spinotrapezius muscle enables study of cellular interactions during healing.(A,B) A 1mm circular volumetric defect made in the spinotrapezius of wildtype mice heals over 7 days, as quantified by

(C)desminimmunostaining. (D) High-powered 3D images show granulation tissue under brightfield microscopy at days 3 and 7 post-injury, coinciding with accumulation of immunostainedCD206+ cells. (E) Revascularization (shown by CD31 immunostaining) occurs 
along the defect edge at day 3 post-injury and among regenerating fibers at day 7. (F) Merged 3D images show the distribution of CD206+ cells among muscle fibers and revascularized muscle tissue. Data presented as mean \pm S.E.M. Statistical analyses were performed using twotailed t-tests. ${ }^{*} \mathrm{p}<0.05, \mathrm{n}=4-5$ animals per group.

Figure 2. Spinotrapezius volumetric muscle loss results in dynamic myeloid cell kinetics at both the systemic and tissue levels.(A)Following volumetric muscle injury in wildtype mice, blood neutrophils peak at day 3 post-injury and return to baseline levels by day 7 (blood neutrophils gated on CD11b+SSC ${ }^{\text {hi }}$ Ly6G $^{\text {hi }}$. . (B) Blood Ly6C ${ }^{\text {hi }}$ monocytes increase over 7 days, while blood Ly6C $\mathrm{C}^{\mathrm{lo}}$ monocytes gradually increase and plateau over days 3-7 (blood monocytes gated on $\mathrm{CD} 11 \mathrm{~b}+\mathrm{SSC}^{\mathrm{lo}} \mathrm{CD} 115+$ ).(C) Neutrophils in injured muscle tissue peak at day 3 and decrease by day 7 post-injury (tissue neutrophils gated on MerTK-CD64-CD11c$\left.\mathrm{CD} 11 \mathrm{~b}+\mathrm{SSC}^{\mathrm{hi}} \mathrm{LyGG}^{\mathrm{hi}}\right)$.(D) $\mathrm{Ly} \mathrm{C}^{\mathrm{hi}}$ monocytes in injured muscle tissue decrease from day 1 to day 7 post-injury, while Ly6C ${ }^{\text {lo }}$ monocytes increase from day 1 to 3 and decrease by day 7 (tissue monocytes gated on CD11b+SSC ${ }^{\text {lo } M e r T K-C D 64-C D 11 c-) .(E) ~ M e r T K+C D 64+~ m a c r o p h a g e s ~}$ increase from day 1 to 7 post-injury. (F) CD206+ macrophages increase from day 1 to 7 postinjury. Data presented as mean \pm S.E.M. Statistical analyses were performed using one-way ANOVA. ${ }^{\dagger} \mathrm{p}<0.05$ compared to Day 0 for Ly6C ${ }^{\mathrm{lo}}$ monocytes or ${ }^{*} \mathrm{p}<0.05$ compared to Day 0 for all other cell types, $n=4-12$ animals per group.

Figure 3. Circulating latex bead-labeled non-classical monocytes are directly recruited to injured spinotrapezius muscle, where they give rise to alternatively activated macrophages.(A) Ly6C ${ }^{\text {lo }}$ monocytes were selectively labeled by intravenous (i.v.) injection of 
latex (LX) beads 1 day prior to VML surgeryin CX3CR1 ${ }^{\mathrm{GFP} /+}$ mice. (B)PLGA thin films were acutely implanted over spinotrapezius VML defects. (C) Both CX3CR $1^{\text {hi }}$ and CX3CR $1^{\text {lo }}$ cells, as well as LX+ cells, are recruited to the defect 3 days post-injury. (D) LX beads co-localize spatially with immunostainedCD68+CD206+ macrophages3 days post-injury. (E) Conditioned media from macrophages stimulated with IL-4 (M(IL-4)) promotes differentiation of C2C12 cells in vitro compared to conditioned media from macrophages stimulated with LPS and IFN- $\gamma$ (M(LPS, IFN- $\gamma))$. Data presented as mean \pm S.D.

Figure 4. On-site delivery of FTY720 increases Ly6C $^{\mathrm{lo}}$, CX3CR1 ${ }^{\text {hi }}$ monocytes in injured muscle.(A)Delivery of FTY720 from PLGA thin films to VML injury in wildtype mice increases the frequency of Ly6C $\mathrm{C}^{\mathrm{lo}}$ monocytes, but not Ly6 $\mathrm{C}^{\mathrm{hi}}$ monocytes3days post-injury compared to unloaded PLGA and no implant controls (tissue monocytes gated on CD11b+SSC $\left.{ }^{\text {lo }}\right)$.(B)Few CX3CR1-GFP+ cells are seen in uninjured, contralateral spinotrapezius muscle in CX3CR1 ${ }^{\mathrm{GFP} /+}$ mice. (C) More CX3CR1 $1^{\text {hi }}$ cells are recruited to peri-defect tissue at day 3 in $\mathrm{CX} 3 \mathrm{CR} 1{ }^{\mathrm{GFP} /+}$ mice treated with FTY720. (D) LX+ cells cluster around defect in FTY720-treated CX3CR1 $1^{\mathrm{GFP} /+}$ mice at day 3 post-injury. Data presented as mean \pm S.E.M. Statistical analyses were performed using two-way ANOVA. * ${ }^{*}<0.05$ compared to Ly6C ${ }^{\text {lo }}$ monocytes in PLGA and no implant controls, $\mathrm{n}=3-4$ animals per group.

Figure 5. FTY720 increases the frequency of CD68+CD206+ cells in injured muscle of CX3CR1 $^{\text {GFP/+ }}$ mice. (A) FTY720-treated micehave a greater proportion of the defect region filled with infiltratingcells at 3 days post-injury, including LX+ and CX3CR1+ cells. (B) Quantification of cellular infiltration in CX3CR1/LX bead/lectin-labeled muscles shows a higher 
percentage of cellular infiltration in defects treated with FTY720. (C) FTY720-treated mice have a greater proportion of the defect region filled withCD68+CD206+ cells compared to PLGA controls 3 days post-injury. (D) Quantification of cellular infiltration in CD68/CD206/CD31 immunostained muscles shows a higher percentage of cellular infiltration in defects treated with FTY720.(E, F) Mice receiving FTY720 have increased density of CD68+CD206+ cells 3 days post-injury. (G) CD68+CD206+ cells are significantly closer to CD31+ blood vessels in the presence of FTY720 3 days post-injury. Data presented as mean \pm S.E.M. Statistical analyses were performed using two-tailed t-tests. ${ }^{*} \mathrm{p}<0.05$, n=3-4 animals per group.

Figure 6. On-site delivery of FTY720 promotes muscle healing 3 days post-injury in CX3CR1 $^{\text {GFP/+ }}$ mice.(A) FTY720-treated mice have increased vascularization in the defect region, as detected by CD31 immunostaining and (B) quantification of CD31+ vessel density. (C) FTY720-treated mice have more collagen deposition within the defect region, as visualized by two-photon microscopy and (D)quantification of the non-collagenous void area.(E)Mice treated with FTY720 have a smaller defect area as measured by desmin immunostaining and (F) quantification of desmin-negative area (dotted red line indicates the mean value of wildtype mice undergoing VML and treated with no implant). Data presented as mean \pm S.E.M. Statistical analyses were performed using two-tailed t-tests. ${ }^{*} \mathrm{p}<0.05$, $\mathrm{n}=3-4$ animals per group.

Figure 7. Local immunomodulation with FTY720 improves muscle repair 7 days postinjury in wildtype mice. (A) FTY720-treated mice have larger and more desmin+ muscle fibers (detected by immunostaining) that more closely recapitulates the structure of an uninjured contralateral control, as well as less dense and aligned collagen (detected by two-photon 
microscopy) compared to PLGA controls. (B) FTY720-treated mice have a reduced volume ratio of collagen to desmin. (C-D) FTY720-treated mice have regenerated muscle fibers with a larger diameter(dotted red line indicates the mean value of wildtype mice undergoing VML and treated with no implant). Data presented as mean \pm S.E.M. Statistical analyses were performed using two-tailed t-tests. ${ }^{*} \mathrm{p}<0.05, \mathrm{n}=3-4$ animals per group. 